\title{
A NEW APPROACH TO LEIBNIZ BIALGEBRAS
}

\author{
ELISABETE BARREIRO AND SAÏD BENAYADI
}

\begin{abstract}
A study of Leibniz bialgebras arising naturally through the double of Leibniz algebras analogue to the classical Drinfeld's double is presented. A key ingredient of our work is the fact that the underline vector space of a Leibniz algebra becomes a Lie algebra and also a commutative associative algebra, when provided with appropriate new products. A special class of them, the coboundary Leibniz bialgebras, gives us the natural framework for studying the Yang-Baxter equation (YBE) in our context, inspired in the classical Yang-Baxter equation as well as in the associative Yang-Baxter equation. Results of the existence of coboundary Leibniz bialgebra on a symmetric Leibniz algebra under certain conditions are obtained. Some interesting examples of coboundary Leibniz bialgebras are also included. The final part of the paper is dedicated to coboundary Leibniz bialgebra structures on quadratic Leibniz algebras.
\end{abstract}

\section{INTRODUCTION}

All the algebras (resp. coalgebras) considered in this paper are finite-dimensional algebras (resp. coalgebras) over a commutative field $\mathbb{K}$ of characteristic zero. A left (resp. right) Leibniz algebra is a nonassociative algebra where the left (resp. right) multiplications are derivations. In 1993 the left (and right) Leibniz algebras were introduced by Jean-Louis Loday [18], as a generalization of Lie algebras with no symmetry requirements. If a nonassociative algebra is both a left Leibniz algebra and a right Leibniz algebra, it is called a symmetric Leibniz algebra [14]. These latter algebras had been considered in [5], appearing in the study of some bi-invariant connections on Lie groups. In recent years, the theory of Leibniz algebras has been intensively studied. Many results of the theory of Lie algebras have been generalized to Leibniz algebras (see for example [7], [9], [10], [13], [14], [15] or [16]). Recently, S. Benayadi and S. Hidri in [6] investigated the structure of left (resp. right) Leibniz algebras endowed with invariant, non-degenerate and symmetric bilinear forms, which are called quadratic left (resp. right) Leibniz algebras. In particular, they prove that a quadratic left (or right) Leibniz algebra is a symmetric Leibniz algebra. It is well known that, in case of Lie theory, double of Lie bialgebras (construction provided by V. Drinfeld [11], see also [8]) are interesting quadratic Lie algebras.

The main purpose of this paper is to explore the concept of Leibniz bialgebra. We show, in particular, that the bialgebra notion of Leibniz can be envisaged only in the case of the symmetric Leibniz algebras. Our approach to Leibniz bialgebras is based on a fundamental result that realizes the underline vector space of the symmetric Leibniz algebra as Lie algebra (resp. commutative associative algebra) with respect to a Lie bracket (resp. commutative associative product) arising in a natural way from the product of the Leibniz algebra. Both Lie algebras and commutative associative algebras have a theory of bialgebras which have been applied to many fields. The Lie bialgebras introduced by Drinfeld [11] arise naturally in the theory of integrable models and in particular are closed related to the classical Yang-Baxter equation. In the case of associative algebras, Joni and Rota in [17] introduced the notion of infinitesimal bialgebra

2010 Mathematics Subject Classification. Primary: 17A32, 17B62; Secondary: 17D25,16W25.

Key words and phrases. Leibniz algebras; representations of Leibniz algebras; Leibniz bialgebras; coboundary Leibniz bialgebras; Lie bialgebras; Classical Yang-Baxter equation; infinitesimal bialgebra; associative Yang-Baxter equation.

This work was partially supported by the Centre for Mathematics of the University of Coimbra - UID/MAT/00324/2013, funded by the Portuguese Government through FCT/MEC and co-funded by the European Regional Development Fund through the Partnership Agreement PT2020. 
in order to provide an algebraic structure for the calculus of divided differences. After that Aguiar developed a systematic theory for infinitesimal bialgebras, based on the analogy with the well developed Lie bialgebra theory $[1,2]$. We develop a bialgebra theory for Leibniz algebras, which acquires a Lie bialgebra structure with respect to the Lie bracket of the Leibniz algebra, and a commutative and cocommutative infinitesimal bialgebra with respect to the commutative associative product of the Leibniz algebra. We also prove that in the setting of the Leibniz algebras there is an analogue of the fundamental classical Drinfeld's double construction in the case of Lie bialgebras. Next, we introduce a Yang-Baxter equation for Leibniz bialgebra, motivated by the classical Yang-Baxter equation (CYBE) and associative Yang-Baxter equation (AYBE). Our approach to Leibniz bialgebras will differ essentially from the approach in the recent work [20] due to A. Rezaei-Aghdam, GH. Haghighatdoost and L. Sedghi-Ghadim, where the authors handle the bialgebras of left (or right) Leibniz algebras by adopting the classic method to study bialgebras for a given nonassociative structure.

The paper is structured as follows. The next section is devoted to review some facts about Leibniz algebras. In Section 3 the notion of Leibniz bialgebra is introduced via the double construction of Leibniz algebras. One of the main results establishes a characterization of the Leibniz bialgebras in terms of coproducts and representations. Section 4 is dedicated to coboundary Leibniz bialgebras, which give us the natural framework for studying the analogue of the classical Yang-Baxter equation (CYBE) and r-matrix in our context, and in Section 5 we establish the existence of coboundary Leibniz bialgebras on a symmetric Leibniz algebra under certain conditions. In Section 6 we included some examples. Finally Section 7 is devoted to the coboundary Lie bialgebras on quadratic Leibniz algebras.

\section{BASIC DEFINITIONS AND RESULTS}

In this section we state some basic definitions and properties concerning Leibniz algebras for convenience of the reader.

Definition 2.1. Let $\mathfrak{L}$ be an algebra endowed with a bilinear multiplication $\mathfrak{L} \times \mathfrak{L} \longrightarrow \mathfrak{L}$.

(i) $\mathfrak{L}$ is called a left Leibniz algebra if

$$
x .(y . z)=(x . y) . z+y \cdot(x . z), \quad \forall x, y, z \in \mathfrak{L} \quad \text { (left Leibniz identity). }
$$

(ii) $\mathfrak{Q}$ is said a right Leibniz algebra if

$$
x .(y . z)=(x . y) \cdot z-(x . z) \cdot y, \quad \forall x, y, z \in \mathbb{L} \quad \text { (right Leibniz identity). }
$$

Definition 2.2. Let $(\mathfrak{L},$.$) be an algebra. Recall that a linear map D: \mathfrak{L} \longrightarrow \mathfrak{L}$ is called a derivation if

$$
D(x \cdot y)=(D x) \cdot y+x \cdot(D y), \quad \forall x, y \in \mathfrak{L} .
$$

As usual, for $x \in \mathfrak{L}$, we define the corresponding endomorphism of $\mathfrak{L}$ by $L_{x}(y)=x \cdot y\left(\right.$ resp. $\left.R_{x}(y)=y \cdot x\right)$, $\forall y \in \mathfrak{L}$, which is called the left (resp. right) multiplication by $x$. We have the following characterization of Leibniz algebras:

(i) $(\mathfrak{L},$.$) is a left (resp. right) Leibniz algebra if the left multiplication L_{x}$ (resp. right multiplication $R_{x}$ ) is a derivation on $\mathfrak{L}$, for any $x \in \mathfrak{Q}$.

(ii) In case that $(\mathfrak{L},$.$) is simultaneously a left and a right Leibniz algebra it is called a symmetric$ Leibniz algebra (or is simply called Leibniz algebra).

It is easy to see that a left (or right) Leibniz algebra $\mathfrak{L}$, such that $x . x=0$ for any $x \in \mathfrak{L}$, is actually a Lie algebra. Conversely, any Lie algebra is both a left and a right Leibniz algebra, meaning that it is a symmetric Leibniz algebra. Although the multiplication in a Leibniz algebra assumes no symmetry property, there is the following weak symmetry condition.

Proposition 2.3. [6] If ( $\mathfrak{Q}$, .) is a left (resp. right) Leibniz algebra then, for any $x, y \in \mathfrak{L}$,

$$
R_{x} L_{y}=-R_{x} R_{y} \quad\left(\text { resp. } L_{x} R_{y}=-L_{x} L_{y}\right) .
$$


If $(\mathfrak{Q},$.$) is a left (resp. right) Leibniz algebra, the condition in Proposition 2.3$ can be written explicitly as

$$
(x \cdot y) \cdot z=-(y \cdot x) \cdot z \quad(\text { resp. } x \cdot(y \cdot z)=-x \cdot(z \cdot y))
$$

for any $x, y, z \in \mathfrak{L}$.

Proposition 2.4. [6] If ( $(\mathfrak{L}$, .) is a symmetric Leibniz algebra then:

$$
\begin{array}{ll}
L_{x} L_{y}=-R_{x} L_{y}, & L_{x . y}=R_{y . x}, \\
R_{x} R_{y}=-L_{x} R_{y}, & L_{x} L_{y}=R_{x} R_{y},
\end{array}
$$

hold for every $x, y \in \mathfrak{R}$.

The conditions for a symmetric Leibniz algebra $\mathfrak{L}$ presented in Proposition 2.4 may be written explicitly as

$$
\begin{aligned}
& x \cdot(y \cdot z)=-(y \cdot z) \cdot x, \quad(x \cdot y) \cdot z=z \cdot(y \cdot x), \\
& (z \cdot y) \cdot x=-x \cdot(z \cdot y), \quad x \cdot(y \cdot z)=(z \cdot y) \cdot x,
\end{aligned}
$$

for every $x, y, z \in \mathfrak{I}$. It is easy to observe that $L_{x} L_{y}=-R_{x} L_{y}$ represents the same as $R_{x} R_{y}=-L_{x} R_{y}$ and also $L_{x . y}=R_{y . x}$ is equal to $L_{x} L_{y}=R_{x} R_{y}$.

Definition 2.5. Let $(\mathfrak{L},$.$) be a nonassociative algebra, V$ a vector space and $r, l: \mathfrak{L} \longrightarrow$ End $(V)$ a pair of linear maps.

(i) If $(\mathfrak{L},$.$) is a left Leibniz algebra, we say that the pair (r, l)$ is a left representation of $\mathfrak{L}$ in $V$ if

$$
\begin{aligned}
& \left(l_{1}\right) l(x . y)=[l(x), l(y)], \\
& \left(l_{2}\right) r(x . y)=r(y) r(x)+l(x) r(y), \\
& \left(l_{3}\right) r(x . y)=l(x) r(y)-r(y) l(x), \quad \forall x, y \in \mathbb{L} .
\end{aligned}
$$

(ii) If $(\mathfrak{L},$.$) is a right Leibniz algebra, we say that (r, l)$ is a right representation of $\mathfrak{L}$ in $V$ if

$$
\begin{aligned}
& \left(r_{1}\right) l(x . y)=[r(y), l(x)], \\
& \left(r_{2}\right) l(x . y)=l(x) l(y)+r(y) l(x), \\
& \left(r_{3}\right) r(x . y)=r(y) r(x)-r(x) r(y), \quad \forall x, y \in \mathfrak{L} .
\end{aligned}
$$

(iii) If $(\mathfrak{L},$.$) is a symmetric Leibniz algebra, we say that (r, l)$ is a representation of $\mathfrak{L}$ in $V$ if it is both a left and a right representation of $\mathfrak{L}$ in $V$. We denote by $\operatorname{Rep}(\mathfrak{L}, V)$ the set of all representations of $\mathfrak{R}$ in $V$.

Remark 2.6. Let $(\mathfrak{L},$.$) be a left (or right) Leibniz algebra. We define the linear map L_{\mathfrak{l}}: \mathfrak{L} \longrightarrow$ End $(\mathfrak{L})$ by $L_{\mathfrak{Q}}(x)=L_{x}$ (resp. $R_{\mathfrak{L}}: \mathfrak{L} \longrightarrow$ End $(\mathfrak{L})$ by $R_{\mathfrak{L}}(x)=R_{x}$ ), for all $x \in \mathfrak{L}$, where we consider the left (resp. right) multiplications on $\mathfrak{L}$. It can be proven that if $\mathfrak{L}$ is a left (resp. right) Leibniz algebra, $\left(R_{\mathfrak{L}}, L_{\mathfrak{I}}\right)$ is a left (resp. right) representation of $\mathfrak{L}$ in $\mathfrak{L}$. In both cases, such a pair $\left(R_{\mathfrak{L}}, L_{\mathfrak{L}}\right)$ is called the adjoint representation of $\mathfrak{Q}$.

If the Leibniz algebra $(\mathfrak{L},$.$) is a Lie algebra, the three conditions of (2.1) (and similar to (2.2)) reduce$ actually to the fact that $L_{\mathfrak{L}}: \mathfrak{L} \longrightarrow$ End ( $\mathfrak{L}$ ) is a Lie algebra homomorphism (because $R_{x}=-L_{x}$ for any $x \in \mathfrak{L})$, so $(r, l)$ is a representation of $(\mathfrak{L},$.$) as a Lie algebra. This suggests that the Definition 2.5$ constitutes a natural generalization of the notion of representation in Lie algebras to our context.

Proposition 2.7. [6] Let $(\mathfrak{Q},$.$) be a symmetric Leibniz algebra, V$ a vector space and $r, l: \mathfrak{L} \longrightarrow$ End $(V)$ a pair of linear maps. Then $(r, l)$ is a representation of $\mathfrak{L}$ in $V$ if and only if,

$$
\begin{array}{cll}
l(x . y)=[l(x), l(y)], & r(x . y)=[l(x), r(y)], & l(x . y)=[r(y), l(x)], \\
l(x) l(y)=-r(x) l(y), & l(x) r(y)=-r(x) r(y), & r(x . y)=-l(x . y),
\end{array}
$$

hold for any $x, y \in \mathfrak{R}$. In this condition, we say that $V$ is an $\mathfrak{L}$-bimodule. 
Remark 2.8. Take ( $\mathfrak{L},$.$) a left (resp. right) Leibniz algebra, V$ a vector space, $V^{*}$ its dual vector space and $r, l: \mathfrak{L} \longrightarrow$ End $(V)$ a left (resp. right) representation of $\mathfrak{L}$ in $V$. In general, the pair of the bilinear maps $l^{*}: \mathfrak{L} \longrightarrow$ End $\left(V^{*}\right)$ and $r^{*}: \mathfrak{L} \longrightarrow$ End $\left(V^{*}\right)$ defined by

$$
\begin{aligned}
& l^{*}(x)(f)=f \circ r(x), \\
& r^{*}(x)(f)=f \circ l(x), \quad \forall x \in \mathfrak{L}, f \in V^{*},
\end{aligned}
$$

is neither a left representation of $\mathfrak{L}$ in $V^{*}$ nor a right representation of $\mathfrak{L}$ in $V^{*}$.

Let $(\mathfrak{L},$.$) be a left (resp. right) Leibniz algebra, \mathfrak{L}^{*}$ its dual vector space and consider $\left(R_{\mathfrak{L}}, L_{\mathfrak{L}}\right)$ the adjoint representation of $\mathfrak{L}$. We define the linear maps $\left(L_{\mathfrak{L}}\right)^{*}: \mathfrak{L} \longrightarrow$ End $\left(\mathfrak{L}^{*}\right)$ and $\left(R_{\mathfrak{L}}\right)^{*}: \mathfrak{L} \longrightarrow$ End $\left(\mathfrak{L}^{*}\right)$ by means of

$$
\begin{aligned}
& \left(L_{\mathfrak{L}}\right)^{*}(x)(f)=L_{x}^{*}(f)=f \circ R_{\mathfrak{L}}(x), \\
& \left(R_{\mathfrak{L}}\right)^{*}(x)(f)=R_{x}^{*}(f)=f \circ L_{\mathfrak{L}}(x), \quad \forall x \in \mathfrak{L}, f \in \mathfrak{L}^{*} .
\end{aligned}
$$

The following result says in which conditions the pair $\left(\left(R_{\mathfrak{L}}\right)^{*},\left(L_{\mathfrak{Q}}\right)^{*}\right)$ is a left (resp. right) representation of $\mathfrak{L}$ in $\mathfrak{Q}^{*}$.

Proposition 2.9. [6] Let ( $\mathfrak{L},$.$) be a left (resp. right) Leibniz algebra. Then the pair \left(\left(R_{\mathfrak{L}}\right)^{*},\left(L_{\mathfrak{L}}\right)^{*}\right)$ is a left (resp. right) representation of $\mathfrak{L}$ in $\mathfrak{L}^{*}$ if and only if $(\mathfrak{L},$.$) is a right (resp. left) Leibniz algebra (i.e., (\mathfrak{L},$. is a symmetric Leibniz algebra). This representation $\left(\left(R_{\mathfrak{L}}\right)^{*},\left(L_{\mathfrak{Q}}\right)^{*}\right)$ is called the coadjoint representation of $\mathfrak{Q}$.

Remark 2.10. Let $(\mathfrak{L},$.$) be a symmetric Leibniz algebra. We easily see that \left(R_{\mathfrak{L}}, L_{\mathfrak{L}}\right)$ is a representation of $\mathfrak{L}$ in $\mathfrak{L}$, so $\mathfrak{L}$ is a $\mathfrak{L}$-bimodule. Applying Proposition 2.9 , we conclude that the pair $\left(\left(R_{\mathfrak{L}}\right)^{*},\left(L_{\mathfrak{L}}\right)^{*}\right)$ is a representation of $\mathfrak{L}$ in $\mathfrak{L}^{*}$, thus $\mathfrak{L}^{*}$ is also a $\mathfrak{L}$-bimodule.

Let $(\mathfrak{L},$.$) be a left (or right) Leibniz algebra. We consider the following two new products on the underline$ vector space:

$$
[x, y]:=\frac{1}{2}(x . y-y \cdot x), \quad x \bullet y:=\frac{1}{2}(x . y+y \cdot x), \quad \forall x, y \in \mathfrak{R} .
$$

Let us denote $\mathfrak{L}^{-}:=(\mathfrak{L},[]$,$) and \mathfrak{I}^{+}:=(\mathfrak{L}, \bullet)$. As usual, the center of $\mathfrak{L}^{-}$is defined by $3\left(\mathfrak{L}^{-}\right):=\{x \in \mathfrak{L}:$ $[x, y]=0, \forall y \in \mathfrak{L}\}$ and the annihilator of $\mathfrak{L}^{+}$is given by $\operatorname{Ann}\left(\mathfrak{L}^{+}\right):=\{x \in \mathfrak{L}: x \bullet y=0, \forall y \in \mathbb{L}\}$. These new products allow the space $\mathfrak{L}$ to become a Lie algebra as well as a commutative associative algebra. The following proposition will be very useful to study Leibniz bialgebras in the sequel.

Proposition 2.11. Consider ( $\mathfrak{Q},$.$) a left (or right) Leibniz algebra. The algebraic structure ( \mathfrak{Q},$.$) is a$ symmetric Leibniz algebra if and only if the following four conditions hold:

(1) $\mathfrak{Q}^{-}$is a Lie algebra;

(2) $\left(\mathfrak{L}^{+}\right)^{3}=\{0\}$;

(3) $\left(\mathfrak{Q}^{+}\right)^{2} \subseteq 3\left(\mathfrak{Q}^{-}\right)$;

(4) $\left[\mathfrak{Q}^{-}, \mathfrak{Q}^{-}\right] \subseteq \operatorname{Ann}\left(\mathfrak{Q}^{+}\right)$.

Proof. By using Proposition 2.4 , we prove by straightforward calculations that if $(\mathfrak{L},$.$) is a symmetric$ Leibniz algebra then the four conditions above are satisfied.

Conversely, suppose that $(\mathfrak{L}$, .) satisfies these four conditions. For $x, y, z \in \mathfrak{L}$, we have

$$
\begin{aligned}
& x .(y . z)=x .[y, z]+x .(y \bullet z)=[x,[y, z]]+x \bullet[y, z]+[x,(y \bullet z)]+x \bullet(y \bullet z)=[x,[y, z]], \\
& (x . y) . z=[x . y, z]+(x . y) \bullet z=[[x, y], z]+[x \bullet y, z]+[x, y] \bullet z+(x \bullet y) \bullet z=[[x, y], z], \\
& y .(x . z)=y .[x, z]+y .(x \bullet z)=[y,[x, z]]+y \bullet[x, z]+[y,(x \bullet z)]+y \bullet(x \bullet z)=[y,[x, z]] .
\end{aligned}
$$

Therefore $x .(y . z)-(x . y) . z-y \cdot(x . z)=[x,[y, z]]+[z,[x, y]]+[y,[z, x]]=0$ because $[$,$] is a Lie structure$ on $\mathfrak{L}$. Now, $(x . y) \cdot z+z \cdot(x . y)=[[x, y], z]+[z,[x, y]]=0$. We conclude that $(\mathfrak{L},$.$) is a symmetric Leibniz$ algebra. 
Definition 2.12. Let $\mathfrak{Q}$ be a Leibniz algebra. A bilinear form $B$ on $\mathfrak{Q}$ is

(i) symmetric if $B(x, y)=B(y, x), \forall x, y \in \mathfrak{L}$;

(ii) non-degenerate if $x \in \mathfrak{L}$ satisfies $B(x, y)=0, \forall y \in \mathfrak{L}$, then $x=0$;

(iii) invariant if $B(x . y, z)=B(x, y \cdot z), \forall x, y, z \in \mathfrak{L}$.

Definition 2.13. A Leibniz algebra $\mathfrak{L}$ is quadratic if there exists a bilinear form $B$ on $\mathfrak{L}$ such that $B$ is symmetric, non-degenerate and invariant. It is denoted by $(\mathfrak{Q}, B)$ and $B$ is called an invariant scalar product on $\mathfrak{Q}$.

\section{LEIBNIZ BIALGEBRAS VIA THE DOUBLE CONSTRUCTION OF A LEIBNIZ ALGEBRA}

The purpose of this section is to introduce the notion of Leibniz bialgebras and to present some of its properties. To start, we recall some definitions on Lie bialgebras and associative bialgebras. A Lie coalgebra is a vector space $\mathfrak{g}$ over $\mathbb{K}$ equipped with a linear map $\Delta_{\mathfrak{l}}: \mathfrak{g} \longrightarrow \mathfrak{g} \otimes \mathfrak{g}$ such that:

$$
\begin{aligned}
& \operatorname{Im}\left(\Delta_{\mathfrak{L}}\right) \subseteq \operatorname{Im}(\mathrm{I}-\tau), \\
& \left(\mathrm{I}+\xi+\xi^{2}\right) \circ\left(\mathrm{I} \otimes \Delta_{\mathfrak{L}}\right) \circ \Delta_{\mathfrak{L}}=0, \quad \text { (co-Jacoby identity) }
\end{aligned}
$$

where I $: \mathfrak{g} \longrightarrow \mathfrak{g}$ represents the identity map, $\tau: \mathfrak{g} \otimes \mathfrak{g} \longrightarrow \mathfrak{g} \otimes \mathfrak{g}$ the linear map (the so called twist map) given by $\tau(x \otimes y)=y \otimes x$ (for any $x, y \in \mathfrak{g}$ ) and $\xi: \mathfrak{g} \otimes \mathfrak{g} \otimes \mathfrak{g} \longrightarrow \mathfrak{g} \otimes \mathfrak{g} \otimes \mathfrak{g}$ denotes the linear cycle map defined by $\xi(x \otimes y \otimes z)=y \otimes z \otimes x$, for all $x, y, z \in \mathfrak{g}$. For more information on Lie coalgebras we refer the reader to [19]. A Lie bialgebra is a vector space $\mathfrak{g}$ over $\mathbb{K}$ together with two linear maps [,] $: \mathfrak{g} \otimes \mathfrak{g} \longrightarrow \mathfrak{g}$ and $\Delta_{\mathfrak{L}}: \mathfrak{g} \longrightarrow \mathfrak{g} \otimes \mathfrak{g}$ such that $(\mathfrak{g},[]$,$) is a Lie algebra, \left(\mathfrak{g}, \Delta_{\mathfrak{L}}\right)$ is a Lie coalgebra and these structures are compatible in the following sense:

$$
\Delta_{\mathfrak{L}}([x, y])=\left(\operatorname{ad}_{x} \otimes \mathrm{I}+\mathrm{I} \otimes \operatorname{ad}_{x}\right) \Delta_{\mathfrak{L}}(y)-\left(\operatorname{ad}_{y} \otimes \mathrm{I}+\mathrm{I} \otimes \operatorname{ad}_{y}\right) \Delta_{\mathfrak{L}}(x),
$$

for any $x, y \in \mathfrak{L}$, where as usual $\operatorname{ad}_{x}(y)=[x, y]$. The compatibility condition can be also expressed as:

$$
\Delta_{\mathfrak{L}}([x, y])=x \cdot \Delta_{\mathfrak{Q}}(y)-y \cdot \Delta_{\mathfrak{Q}}(x),
$$

for any $x, y \in \mathfrak{g}$, where the tensor product $\mathfrak{g} \otimes \mathfrak{g}$ is a $\mathfrak{g}$-module via the adjoint action defined by:

$$
x \cdot\left(\sum_{i=1}^{n} x_{i} \otimes y_{i}\right)=\sum_{i=1}^{n}\left(\left[x, x_{i}\right] \otimes y_{i}+x_{i} \otimes\left[x, y_{i}\right]\right),
$$

for any $x, x_{i}, y_{i} \in \mathfrak{g}$ with $i \in\{1, \ldots, n\}$ (i.e., $\Delta_{\mathfrak{l}}$ is a 1-cocycle of $\mathfrak{g}$ with values in $\mathfrak{g} \otimes \mathfrak{g}$, so $\Delta_{\mathfrak{l}} \in \mathrm{Z}^{1}(\mathfrak{g}, \mathfrak{g} \otimes \mathfrak{g})$ ). The reader may consult [8] for a survey of results on Lie bialgebras.

If $A$ is a $\mathbb{K}$-vector space provided with a linear map $\Delta_{a}: A \longrightarrow A \otimes A$, the pair $\left(A, \Delta_{a}\right)$ is called coassociative coalgebra if the following condition holds:

$$
\left(\mathrm{I} \otimes \Delta_{a}\right) \circ \Delta_{a}=\left(\Delta_{a} \otimes \mathrm{I}\right) \circ \Delta_{a} \quad \text { (co-associativity identity). }
$$

Let $(A, \bullet)$ be an associative algebra provided with a linear map $\Delta_{a}: A \longrightarrow A \otimes A$, the triple $\left(A, \bullet, \Delta_{a}\right)$ is called infinitesimal bialgebra if $\left(A, \Delta_{a}\right)$ is a coassociative coalgebra and

$$
\Delta_{a}(x \bullet y)=(L(x) \otimes \mathrm{I}) \Delta_{a}(y)+(\mathrm{I} \otimes R(y)) \Delta_{a}(x),
$$

for any $x, y \in A$, where $L(x)$ is the left multiplication by $x$ and $R(y)$ is the right multiplication by $y$ in the associative algebra $(A, \bullet)$. See $[1,2]$ for additional information about infinitesimal bialgebras.

We approach the notion of a Leibniz bialgebra in a similar way to Lie bialgebra and infinitesimal bialgebra notions. Let $(\mathfrak{L},$.$) be a left (resp. right) Leibniz algebra and define m: \mathfrak{L} \otimes \mathfrak{L} \longrightarrow \mathfrak{L}$ by $m(x \otimes y):=x . y$, for any $x, y \in \mathfrak{L}$. The condition in definition of left (resp. right) Leibniz algebra may be written as:

$$
\begin{aligned}
m \circ(\mathrm{I} \otimes m) & =m \circ(m \otimes \mathrm{I})+m \circ(\mathrm{I} \otimes m) \circ(\tau \otimes \mathrm{I}) \\
(\text { resp. } & m \circ(m \otimes \mathrm{I})=m \circ(\mathrm{I} \otimes m)+m \circ(\mathrm{I} \otimes m) \circ(\mathrm{I} \otimes \tau)) .
\end{aligned}
$$


The following notion of left (resp. right) Leibniz coalgebra is naturally deduced from the definition of left (resp. right) Leibniz algebra using $m$.

Definition 3.1. Assume that $\mathfrak{Q}$ is a vector space provided with a linear comultiplication $\Delta: \mathfrak{L} \longrightarrow \mathfrak{L} \otimes \mathfrak{L}$.

(i) We say that $\mathfrak{Q}$ is a left Leibniz coalgebra if

$$
(\mathrm{I} \otimes \Delta) \circ \Delta=(\Delta \otimes \mathrm{I}) \circ \Delta+(\tau \otimes \mathrm{I}) \circ(\mathrm{I} \otimes \Delta) \circ \Delta \quad \text { (left co-Leibniz identity). }
$$

(ii) We call that $\mathfrak{Q}$ is a right Leibniz coalgebra if

$$
(\Delta \otimes \mathrm{I}) \circ \Delta=(\mathrm{I} \otimes \Delta) \circ \Delta+(\mathrm{I} \otimes \tau) \circ(\Delta \otimes \mathrm{I}) \circ \Delta \quad \text { (right co-Leibniz identity) }
$$

(iii) If $\mathfrak{L}$ is both a left and right Leibniz coalgebra it is called a symmetric Leibniz coalgebra (or simply-Leibniz coalgebra).

For the comultiplication of $x$ in $\mathfrak{L}$, we write $\Delta(x)=\sum_{x} x_{(1)} \otimes x_{(2)}$, where $x_{(1)}$ and $x_{(2)}$ are elements in $\mathfrak{L}$. We use the notation: if $\mathfrak{B}$ is a vector space, $\mathfrak{B}^{*}$ its dual, $x \in \mathfrak{B}$ and $f \in \mathfrak{B}^{*}$, we denote $f(x)$ by either $\langle f, x\rangle$ or $\langle x, f\rangle$. Moreover, we identify $\mathfrak{B}$ and its bi-dual $\mathfrak{B}^{* *}$ by means the isomorphism of vector spaces $\mathfrak{B} \rightarrow \mathfrak{B}^{* *}$ given by $x \mapsto\langle x$,$\rangle .$

There is a natural way to define a structure of a Leibniz coalgebra on the dual of a Leibniz algebra. Let $(\mathfrak{L}, m:=$.) be a left (or right) Leibniz algebra, and on the dual space of $\mathfrak{L}$ we consider the linear map ${ }^{t} m: \mathfrak{L}^{*} \longrightarrow \mathfrak{L}^{*} \otimes \mathfrak{Q}^{*}$ defined by

$$
\left\langle{ }^{t} m(f), x \otimes y\right\rangle=\langle f, m(x \otimes y)\rangle, \quad \forall x, y \in \mathfrak{L}, f \in \mathfrak{Q}^{*} .
$$

It is easy to check that $(\mathfrak{L}, m)$ is a left (resp. right) Leibniz algebra if and only if $\left(\mathfrak{I}^{*},{ }^{t} m\right)$ is a left (resp. right) Leibniz coalgebra.

In the other way around, we also endow the dual of a Leibniz coalgebra with a structure of a Leibniz algebra. Let $(\mathfrak{L}, \Delta)$ be a left (resp. right) Leibniz coalgebra. It turns out that $\left(\mathfrak{L}^{*}, \star\right)$ is a left (resp. right) Leibniz algebra, where $\star$ is defined by:

$$
\langle f \star g, x\rangle:=\langle f \otimes g, \Delta(x)\rangle, \quad \forall f, g \in \mathfrak{Q}^{*}, x \in \mathfrak{L} .
$$

Now, we are in a position to construct the double of $(\mathfrak{L}, ., \Delta)$ such that $(\mathfrak{L},$.$) is a left (resp. right) Leibniz$ algebra and $(\mathfrak{L}, \Delta)$ is a left (resp. right) Leibniz coalgebra. On the vector space $\mathcal{D}(\mathfrak{L}):=\mathfrak{L} \oplus \mathfrak{L}^{*}$, we consider the following product:

$$
(x, f) \odot(y, g):=\left(x \cdot y+\left(R_{\mathfrak{L}^{*}}\right)^{*}(g)(x)+\left(L_{\mathfrak{Q}^{*}}\right)^{*}(f)(y), f \star g+\left(R_{\mathfrak{L}}\right)^{*}(y)(f)+\left(L_{\mathfrak{L}}\right)^{*}(x)(g)\right),
$$

for all $x, y \in \mathfrak{L}, f, g \in \mathfrak{Q}^{*}$. It is clear that if $(\mathcal{D}(\mathfrak{L}), \odot)$ is a left (resp. right) Leibniz algebra, then the pair $\left(\left(R_{\mathfrak{L}}\right)^{*},\left(L_{\mathfrak{L}}\right)^{*}\right)$ is a left (resp. right) representation of $(\mathfrak{L},$.$) in \mathfrak{L}^{*}$ and the pair $\left(\left(R_{\mathfrak{L}^{*}}\right)^{*},\left(L_{\mathfrak{Q}^{*}}\right)^{*}\right)$ is a left (resp. right) representation of $\left(\mathfrak{L}^{*}, \star\right)$ in $\mathfrak{L} \cong \mathfrak{L}^{* *}$. Consequently, by Proposition 2.9 , we have that $(\mathfrak{L},$.$) and$ $\left(\mathfrak{Q}^{*}, \star\right)$ are symmetric Leibniz algebras, so $(\mathfrak{L}, \Delta)$ is also a symmetric Leibniz coalgebra. Let us remark that if $(\mathcal{D}(\mathfrak{L}), \odot)$ is a left (or right) Leibniz algebra, then the symmetric bilinear $B$ form on $\mathcal{D}(\mathfrak{L})$ defined by:

$$
B((x, f),(y, g)):=g(x)+f(y), \quad \forall x, y \in \mathfrak{L}, f, g \in \mathfrak{L}^{*},
$$

is non-degenerate and associative (or invariant). Then $(\mathcal{D}(\mathfrak{L}), \odot$ ) is a symmetric Leibniz algebra by [6]. The above reasoning leads us to introduce the following key definition of Leibniz bialgebra via the notion of the double of a Leibniz algebra, just to Leibniz algebras with symmetric structures:

Definition 3.2. Let us consider $(\mathfrak{L}, ., \Delta)$ such that $(\mathfrak{L},$.$) is a symmetric Leibniz algebra and (\mathfrak{L}, \Delta)$ is symmetric Leibniz coalgebra. The triple $(\mathfrak{L}, ., \Delta)$ is said symmetric Leibniz bialgebra (or simply Leibniz bialgebra $)$ if $(\mathcal{D}(\mathfrak{L}), \odot)$ is a symmetric Leibniz algebra. 
From now on we consider $(\mathfrak{L}, ., \Delta)$ such that $(\mathfrak{L},$.$) is a symmetric Leibniz algebra and (\mathfrak{L}, \Delta)$ is symmetric Leibniz coalgebra (i.e., $\left(\mathfrak{Q}^{*}, \star\right)$ is a symmetric Leibniz algebra). Let us consider the products $[$,$] and \bullet$ on $\mathcal{D}(\mathfrak{L})$ defined by: $\forall x, y \in \mathfrak{L}, f, g \in \mathfrak{L}^{*}$,

$$
\begin{aligned}
{[(x, f),(y, g)]: } & :=\frac{1}{2}((x, f) \odot(y, g)-(y, g) \odot(x, f)) ; \\
(x, f) \bullet(y, g): & :=\frac{1}{2}((x, f) \odot(y, g)+(y, g) \odot(x, f)) .
\end{aligned}
$$

We consider also the products $[,]_{1}, \bullet_{1}$ on $\mathfrak{L}$, and the products $[,]_{2}, \bullet_{2}$ on $\mathfrak{L}^{*}$ given by: $\forall x, y \in \mathfrak{L}, f, g \in$ $\mathfrak{2}^{*}$,

$$
\begin{aligned}
{[x, y]_{1}:=\frac{1}{2}(x . y-y . x) \quad \text { and } \quad x \bullet_{1} y: } & :=\frac{1}{2}(x . y+y \cdot x) ; \\
{[f, g]_{2}:=\frac{1}{2}(f \star g-g \star f) \quad \text { and } \quad f \bullet_{2} g } & :=\frac{1}{2}(f \star g+g \star f) .
\end{aligned}
$$

By Proposition 2.11 , it turns out that the resulting algebraic structure $\mathfrak{L}^{-}:=\left(\mathfrak{L},[,]_{1}\right)\left(\right.$ resp. $\left(\mathfrak{L}^{*}\right)^{-}:=$ $\left.\left(\mathfrak{L}^{*},[,]_{2}\right)\right)$ is a Lie algebra and $\mathfrak{L}^{+}:=\left(\mathfrak{L}, \bullet_{1}\right)\left(\operatorname{resp} .\left(\mathfrak{L}^{*}\right)^{+}:=\left(\mathfrak{L}^{*}, \bullet_{2}\right)\right)$ is a commutative associative algebra such that:

$$
\left(\mathfrak{L}^{+}\right)^{3}=\{0\},\left(\mathfrak{Q}^{+}\right)^{2} \subseteq 3\left(\mathfrak{L}^{-}\right) \text {and }\left[\mathfrak{Q}^{-}, \mathfrak{Q}^{-}\right] \subseteq \operatorname{Ann}\left(\mathfrak{L}^{+}\right)
$$

$$
\left(\text { resp. }\left(\left(\mathfrak{L}^{*}\right)^{+}\right)^{3}=\{0\},\left(\left(\mathbb{L}^{*}\right)^{+}\right)^{2} \subseteq 3\left(\left(\mathfrak{L}^{*}\right)^{-}\right) \text {and }\left[\left(\mathfrak{L}^{*}\right)^{-},\left(\mathfrak{Q}^{*}\right)^{-}\right] \subseteq \operatorname{Ann}\left(\left(\mathfrak{Q}^{*}\right)^{+}\right)\right) .
$$

Consider $\Delta_{\mathfrak{l}}: \mathfrak{L} \longrightarrow \mathfrak{L} \otimes \mathfrak{L}$ and $\Delta_{a}: \mathfrak{L} \longrightarrow \mathfrak{L} \otimes \mathfrak{L}$ defined by, respectively

$$
\begin{aligned}
& \left\langle\Delta_{\mathfrak{Q}}(x), f \otimes g\right\rangle=\left\langle x,[f, g]_{2}\right\rangle \\
& \left\langle\Delta_{a}(x), f \otimes g\right\rangle=\left\langle x, f \bullet_{2} g\right\rangle, \quad \forall x \in \mathfrak{L}, f, g \in \mathbb{R}^{*} .
\end{aligned}
$$

Thus $\left(\mathfrak{L}, \Delta_{\mathfrak{L}}\right)$ is a Lie coalgebra and $\left(\mathfrak{L}, \Delta_{a}\right)$ is a coassociative coalgebra which is cocommutative. We observe that:

$$
\Delta_{\mathfrak{L}}:=\frac{1}{2}(\Delta-\tau \circ \Delta) \text { and } \Delta_{a}:=\frac{1}{2}(\Delta+\tau \circ \Delta) .
$$

Let us fix the notation $\Delta_{\mathfrak{L}}(x)=\sum_{(x)_{\mathfrak{I}}} x_{(1)} \otimes x_{(2)}$ and $\Delta_{a}(x)=\sum_{(x)_{a}} x_{(1)} \otimes x_{(2)}$, for any $x \in \mathfrak{L}$, where $x_{(1)}$ and $x_{(2)}$ are elements of $\mathfrak{L}$. By using these notations and applying Proposition 2.11, we get the following result.

Proposition 3.3. Let $(\mathfrak{L}, \Delta)$ be a left (or right) Leibniz coalgebra. The pair $(\mathfrak{Q}, \Delta)$ is a symmetric Leibniz coalgebra if and only if the following four conditions hold:

(1) $\left(\mathfrak{L}, \Delta_{\mathfrak{L}}\right)$ is a Lie coalgebra;

(2) $\left(\Delta_{a} \otimes I\right) \circ \Delta_{a}=\left(I \otimes \Delta_{a}\right) \circ \Delta_{a}=0$;

(3) $\left(\Delta_{a} \otimes I\right) \circ \Delta_{\mathfrak{L}}=\left(I \otimes \Delta_{a}\right) \circ \Delta_{\mathfrak{L}}=0$;

(4) $\left(\Delta_{\mathfrak{L}} \otimes I\right) \circ \Delta_{a}=\left(I \otimes \Delta_{\mathfrak{L}}\right) \circ \Delta_{a}=0$.

Making use of $\tau \circ \Delta_{a}=\Delta_{a}$ and $\tau \circ \Delta_{\mathfrak{l}}=-\Delta_{\mathfrak{l}}$ we obtain the following consequence

Corollary 3.4. The pair $(\mathfrak{L}, \Delta)$ is a symmetric Leibniz coalgebra if and only if $\left(\mathfrak{L}, \Delta_{\mathfrak{L}}\right)$ is a Lie coalgebra and

$$
\left(\Delta_{a} \otimes I\right) \circ \Delta_{a}=\left(\Delta_{a} \otimes I\right) \circ \Delta_{\mathfrak{L}}=\left(\Delta_{\mathfrak{L}} \otimes I\right) \circ \Delta_{a}=0 .
$$

It is straightforward to verify that $(\mathcal{D}(\mathfrak{L}))^{-}:=(\mathcal{D}(\mathfrak{L}),[]$,$) is a Lie algebra if and only if \left(\mathfrak{L},[,]_{1}, \Delta_{\mathfrak{L}}\right)$ is a Lie bialgebra, which means:

$$
\Delta_{\mathfrak{Q}}\left([x, y]_{1}\right)=\left(\operatorname{ad}_{x} \otimes \mathrm{I}+\mathrm{I} \otimes \operatorname{ad}_{x}\right) \Delta_{\mathfrak{L}}(y)-\left(\operatorname{ad}_{y} \otimes \mathrm{I}+\mathrm{I} \otimes \operatorname{ad}_{y}\right) \Delta_{\mathfrak{L}}(x),
$$

for any $x, y \in \mathfrak{L}, \operatorname{here}_{x}(y)=[x, y]_{1}$. 
We fix the following notation: If $x \in \mathfrak{L}$ (resp. $f \in \mathfrak{L}^{*}$ ), we denote by $\mathbf{M}_{x}$ (resp. $\mathbf{M}_{f}$ ) the endomorphism of $\mathfrak{L}\left(\right.$ resp. $\left.\mathfrak{Q}^{*}\right)$ defined by: $\mathbf{M}_{x}(y):=x \bullet_{1} y, \quad \forall y \in \mathfrak{L}\left(\right.$ resp. $\left.\mathbf{M}_{f}(g):=f \bullet_{2} g, \quad \forall g \in \mathfrak{Q}^{*}\right)$. With the notation introduced above, the products [, ] and $\bullet$ on $\mathcal{D}(\mathfrak{L})$ are also expressed as:

$$
\begin{gathered}
{[(x, f),(y, g)]=\left([x, y]_{1}+x \circ \mathrm{ad}_{g}-y \circ \mathrm{ad}_{f},[f, g]_{2}+f \circ \mathrm{ad}_{y}-g \circ \mathrm{ad}_{x}\right),} \\
(x, f) \bullet(y, g)=\left(x \bullet{ }_{1} y+x \circ \mathrm{M}_{g}+y \circ \mathbf{M}_{f}, f \bullet 2 g+f \circ \mathbf{M}_{y}+g \circ \mathbf{M}_{x}\right),
\end{gathered}
$$

for any $x, y \in \mathfrak{L}, f, g \in \mathfrak{L}^{*}$. Recall that the linear map $\pi: \mathfrak{L} \longrightarrow \operatorname{gl}(\mathfrak{L} \otimes \mathfrak{L})$ defined by $\pi(x)(y \otimes z):=$ $x \cdot \mathfrak{Q}(y \otimes z)-(y \otimes z) \cdot \mathfrak{L} x$, for all $x \in \mathfrak{L}, y \otimes z \in \mathfrak{L} \otimes \mathfrak{L}$, where

$$
\begin{aligned}
& x \cdot \mathfrak{I}(y \otimes z)=\operatorname{ad}_{x}(y) \otimes z=[x, y]_{1} \otimes z, \\
& (y \otimes z) \cdot \mathfrak{Q} x=-y \otimes \operatorname{ad}_{x}(z)=y \otimes[z, x]_{1},
\end{aligned}
$$

is a representation of Lie algebra $\left(\mathfrak{L},[,]_{1}\right)$ on $\mathfrak{L} \otimes \mathfrak{L}$. And also $\mathfrak{L}$ acts on $\mathfrak{L} \otimes \mathfrak{L}$ via the commutative associative regular representation, meaning that

$$
\begin{aligned}
& x \cdot a(y \otimes z)=\mathrm{M}_{x}(y) \otimes z=(x \bullet 1 y) \otimes z, \\
& (y \otimes z){ }_{a} x=y \otimes \mathrm{M}_{x}(z)=y \otimes\left(z \bullet{ }_{1} x\right), \quad \forall x, y, z \in \mathbb{R} .
\end{aligned}
$$

We note that $\mathfrak{L} \otimes \mathfrak{L}$ with these actions is a bimodule of the associative algebra $\left(\mathfrak{L}, \bullet_{1}\right)$. Next results present conditions (2), (3) and (4) presented in Proposition 2.11 in terms of coproducts and representations.

Proposition 3.5. The following assertions are equivalent:

(1) $\left((\mathcal{D}(\mathfrak{Q}))^{+}\right)^{3}=\{0\}$;

(2) For any $x, y \in \mathfrak{L}$ and $f, g \in \mathfrak{L}^{*}$,

$$
\begin{aligned}
& (f \bullet 2 g)(x \bullet 1, y)=0, \\
& f \circ M_{\left(\left\langle x,>M_{g}\right)\right.}=-f \bullet_{2}\left(g \circ M_{x}\right) ;
\end{aligned}
$$

(3) For any $x, y \in \mathfrak{L}$,

$$
\begin{aligned}
& \Delta_{a}(x \bullet 1)=0, \\
& y \cdot{ }_{a} \Delta_{a}(x)+\Delta_{a}(y) \cdot{ }_{a} x=0 .
\end{aligned}
$$

Proof. First we prove (1) $\Longrightarrow(2)$. Assume that $\bullet$ is 3-nilpotent. It is enough to see the cases $(x \bullet y) \bullet f=0$, $x \bullet(f \bullet g)=0, f \bullet(x \bullet g)=0$ and $x \bullet(y \bullet f)=0$, with $x, y \in \mathbb{L}$ and $f, g \in \mathbb{L}^{*}$.

First we study the case $(x \bullet y) \bullet f=0$. Since

$$
(x \bullet y) \bullet f=(x \bullet 1, y) \bullet f=(x \bullet 1, y) \circ \mathbf{M}_{f}+f \circ \mathbf{M}_{x \bullet 1},
$$

from $(x \bullet y) \bullet f=0$ we get $(x \bullet 1, y) \circ \mathbf{M}_{f}=0$ and $f \circ \mathbf{M}_{x \bullet 1 y}=0$. But $f \circ \mathbf{M}_{x \bullet 1 y}(z)=f\left(\left(x \bullet \bullet_{1} y\right) \bullet{ }_{1} z\right)=0$, for $z \in \mathfrak{L}$, by hypothesis. And $\left(x \bullet_{1} y\right) \circ \mathbf{M}_{f}(g)=\left(x \bullet_{1} y\right)\left(f \bullet_{2} g\right)=\left(f \bullet_{2} g\right)(x \bullet 1 y)$, for $g \in \mathfrak{L}^{*}$. It turns out that $(f \bullet 2 g)(x \bullet 1)=0$, getting condition (3.5).

From $x \bullet(f \bullet g)=0$ we obtain the same condition as before. Next, we study the case $f \bullet(x \bullet g)=0$. As

$$
f \bullet(x \bullet g)=f \bullet\left(x \circ \mathrm{M}_{g}+g \circ \mathrm{M}_{x}\right)=x \circ \mathrm{M}_{g} \circ \mathrm{M}_{f}+f \circ \mathrm{M}_{\left(\left\langle x,>\mathrm{M}_{g}\right)\right.}+f \bullet \bullet_{2}\left(g \circ \mathrm{M}_{x}\right),
$$

then using $f \bullet(x \bullet g)=0$ it follows $x \circ \mathrm{M}_{g} \circ \mathrm{M}_{f}=0$ and $f \circ \mathrm{M}_{\left(\langle x,\rangle \circ \mathrm{M}_{g}\right)}+f \bullet 2\left(g \circ \mathrm{M}_{x}\right)=0$. But $x \circ \mathbf{M}_{g} \circ \mathbf{M}_{f}(h)=x\left(g \bullet_{2}(f \bullet 2 h)=0\right.$, for $h \in \mathfrak{R}^{*}$, by hypothesis. So $f \circ \mathbf{M}_{\left(\left\langle x,>\mathbf{M}_{8}\right)\right.}=-f \bullet_{2}\left(g \circ \mathbf{M}_{x}\right)$, obtaining condition (3.6).

We are left to deal with $x \bullet(y \bullet f)=0$. As

$$
x \bullet(y \bullet f)=x \bullet\left(y \circ \mathbf{M}_{f}+f \circ \mathbf{M}_{y}\right)=x \bullet 1\left(y \circ \mathbf{M}_{f}\right)+x \circ \mathbf{M}_{f \circ \mathbf{M}_{y}}+\left(f \circ \mathbf{M}_{y}\right) \circ \mathbf{M}_{x},
$$

from $x \bullet(y \bullet f)=0$ we infer $x \bullet 1\left(y \circ \mathbf{M}_{f}\right)+x \circ \mathbf{M}_{f \circ \mathbf{L}_{y}}=0$ and $\left(f \circ \mathbf{M}_{y}\right) \circ \mathbf{M}_{x}=0$. However $\left(f \circ \mathbf{M}_{y}\right) \circ \mathbf{M}_{x}(z)=$ $f(y \bullet 1(x \bullet 1))=0$, for $z \in \mathfrak{L}$, by hypothesis. The last remaining condition $x \bullet 1\left(y \circ \mathbf{M}_{f}\right)=-x \circ \mathbf{M}_{f \circ \mathbf{M}_{y}}$, 
is equivalent to condition (3.6) already obtained. Indeed, for $y \in \mathfrak{R}, f \circ \mathrm{M}_{x \circ \mathrm{M}_{g}}(y)=f\left(y \bullet_{1}\left(x \circ \mathrm{M}_{g}\right)\right)$ and on the other hand

$$
f \bullet_{2}\left(g \circ \mathbf{M}_{x}\right)(y)=y\left(f \bullet 2\left(g \circ \mathbf{M}_{x}\right)\right)=y \circ \mathbf{M}_{g \circ \mathbf{M}_{x}}(f)=f\left(y \circ \mathbf{M}_{g \circ \mathbf{M}_{x}}\right),
$$

as required.

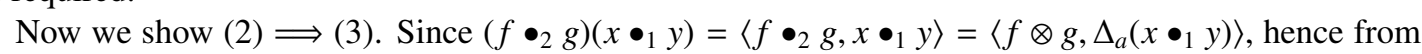
$(f \bullet 2 g)(x \bullet 1, y)=0$ we conclude that $\Delta_{a}(x \bullet 1, y)=0$ getting condition (3.7). Using $\Delta_{a}=\tau \circ \Delta_{a}$

$$
\begin{aligned}
& \left\langle f \circ \mathbf{M}_{\left(\langle x,\rangle \circ \mathbf{M}_{g}\right)}, y\right\rangle=\left\langle f,\left(x \circ \mathbf{M}_{g}\right) \bullet{ }_{1} y\right\rangle=\left\langle\Delta_{a}(f),\left(x \circ \mathbf{M}_{g}\right) \otimes y\right\rangle \\
& =\left\langle\sum_{(f)_{a}} f_{(1)} \otimes f_{(2)},\left(x \circ \mathbf{M}_{g}\right) \otimes y\right\rangle=\sum_{(f)_{a}}\left\langle f_{(1)}, x \circ \mathbf{M}_{g}\right\rangle\left\langle f_{(2)}, y\right\rangle \\
& =\sum_{(f)_{a}}\left\langle x, g \bullet_{2} f_{(1)}\right\rangle\left\langle y, f_{(2)}\right\rangle=\sum_{(f)_{a}}\left\langle\Delta_{a}(x), g \otimes f_{(1)}\right\rangle\left\langle y, f_{(2)}\right\rangle \\
& =\left\langle\Delta_{a}(x) \otimes y, \sum_{(f)_{a}} g \otimes f_{(1)} \otimes f_{(2)}\right\rangle=\left\langle\Delta_{a}(x) \otimes y, g \otimes \Delta_{a}(f)\right\rangle \\
& =\left\langle\Delta_{a}(x) \otimes y,\left(I \otimes \Delta_{a}\right) \circ \tau(f \otimes g)\right\rangle=\left\langle{ }^{t} \tau \circ\left(I \otimes \bullet_{1}\right)\left(\Delta_{a}(x) \otimes y\right), f \otimes g\right\rangle \\
& =\left\langle\sum_{(x)_{a}}\left(x_{(2)} \bullet 1 y\right) \otimes x_{(1)}, f \otimes g\right\rangle=\left\langle y \cdot{ }_{a}\left(\tau \circ \Delta_{a}(x)\right), f \otimes g\right\rangle \\
& =\left\langle y \cdot{ }_{a} \Delta_{a}(x), f \otimes g\right\rangle \\
& \left\langle f \bullet_{2}\left(g \circ \mathbf{M}_{x}\right), y\right\rangle=\left\langle f \otimes\left(g \circ \mathbf{M}_{x}\right), \Delta_{a}(y)\right\rangle=\left\langle f \otimes\left(g \circ \mathbf{M}_{x}\right), \sum_{(y)_{a}} y_{(1)} \otimes y_{(2)}\right\rangle \\
& =\sum_{(y)_{a}}\left\langle f, y_{(1)}\right\rangle\left\langle g, x \bullet{ }_{1} y_{(2)}\right\rangle=\left\langle f \otimes g, \sum_{(y)_{a}} y_{(1)} \otimes\left(x \bullet_{1} y_{(2)}\right)\right\rangle \\
& =\left\langle f \otimes g, \Delta_{a}(y) \cdot{ }_{a} x\right\rangle
\end{aligned}
$$

Therefore, from $f \circ \mathbf{M}_{\left(\langle x,\rangle \circ \mathbf{M}_{g}\right)}=-f \bullet \bullet_{2}\left(g \circ \mathbf{M}_{x}\right)$ we conclude that $y \cdot{ }_{a} \Delta_{a}(x)+\Delta_{a}(y) \cdot{ }_{a} x=0$ as required. The converse of these two implications are clearly true, we just have to make the reasonings above in the opposite direction, so this show the desired equivalences.

Proposition 3.6. The following statements are equivalent:

(1) $\left((\mathcal{D}(\mathfrak{L}))^{+}\right)^{2} \subseteq 3\left((\mathcal{D}(\mathfrak{Q}))^{-}\right)$;

(2) For any $x, y \in \mathfrak{L}$ and $f, g \in \mathfrak{L}^{*}$,

$$
\begin{aligned}
& {[f, g]_{2}\left(x \bullet \bullet_{1} y\right)=0,} \\
& (f \bullet 2)[x, y]_{1}=0, \\
& g \circ a d_{\left(\left\langle x,>M_{f}\right)\right.}=\left[f \circ M_{x}, g\right]_{2} ;
\end{aligned}
$$

(3) For any $x, y \in \mathfrak{R}$,

$$
\begin{aligned}
& \Delta_{\mathfrak{Q}}(x \bullet 1, y)=0, \\
& \Delta_{a}\left([x, y]_{1}\right)=0, \\
& x \cdot{ }_{a} \Delta_{\mathfrak{Q}}(y)-\Delta_{a}(x) \cdot \mathfrak{g} y=0 .
\end{aligned}
$$

Proof. First we show (1) $\Longrightarrow(2)$. We have to study three cases, depending on the choice of the two elements in $\mathfrak{L} \cup \mathfrak{L}^{*}$. First, we take $x, y \in \mathfrak{L}$. Since $\mathfrak{L} \bullet \mathfrak{L} \subseteq Z\left(\mathfrak{L}^{-}\right)$then it is enough to take $f \in \mathfrak{L}^{*}$. We have

$$
[x \bullet y, f]=(x \bullet 1, y) \circ \operatorname{ad}_{f}-f \circ \operatorname{ad}_{x_{1} y},
$$


from $[x \bullet y, f]=0$ we obtain $\left(x \bullet_{1} y\right) \circ \operatorname{ad}_{f}=0$ and $f \circ \operatorname{ad}_{x \bullet 1}=0$. But $f \circ \operatorname{ad}_{x \bullet_{1} y}(z)=f\left(\left[x \bullet \bullet_{1} y, z\right]_{1}\right)=0$, for $z \in \mathfrak{L}$, by hypothesis. And $\left(x \bullet_{1} y\right) \circ \operatorname{ad}_{f}(g)=[f, g]_{2}\left(x \bullet \bullet_{1} y\right)$, for $g \in \mathfrak{Q}^{*}$. So $[f, g]_{2}\left(x \bullet \bullet_{1} y\right)=0$, getting condition (3.9).

Now, we take $f, g \in \mathfrak{L}^{*}$. Since $\mathfrak{Q}^{*} \bullet \mathfrak{Q}^{*} \subseteq Z\left(\left(\mathfrak{L}^{*}\right)^{-}\right)$then we just take $x \in \mathfrak{L}$. We have

$$
[f \bullet g, x]=-x \circ \operatorname{ad}_{f \bullet 2 g}+(f \bullet 2 g) \circ \operatorname{ad}_{x} .
$$

Using the fact that $[f \bullet g, x]=0$ we obtain $x \circ \operatorname{ad}_{f \bullet 2 g}=0$ and $\left(f \bullet_{2} g\right) \circ \operatorname{ad}_{x}=0$. But $x \circ \operatorname{ad}_{f \bullet_{2} g}(h)=$ $x\left(\left[f \bullet_{2} g, h\right]_{2}\right)=0$, for $h \in \mathfrak{L}^{*}$, by hypothesis. And $\left(f \bullet_{2} g\right) \circ \operatorname{ad}_{x}(y)=\left(f \bullet_{2} g\right)[x, y]_{1}$, for $y \in \mathfrak{L}$. Thus $\left(f \bullet_{2} g\right)[x, y]_{1}=0$, getting condition (3.10).

Finally, we take $x \in \mathfrak{Q}$ and $f \in \mathfrak{Q}^{*}$. For $g \in \mathfrak{Q}^{*}$ we get

$\left.[x \bullet f, g]=\left[x \circ \mathbf{M}_{f}+f \circ \mathbf{M}_{x}, g\right]=\left[x \circ \mathbf{M}_{f}, g\right]+\left[f \circ \mathbf{M}_{x}, g\right]=\left(x \circ \mathbf{M}_{f}\right) \circ \operatorname{ad}_{g}-g \circ \operatorname{ad}_{(\langle x,\rangle} \mathbf{M}_{f}\right)+\left[f \circ \mathbf{M}_{x}, g\right]_{2}$

from $[x \bullet f, g]=0$ it follows $\left(x \circ \mathbf{M}_{f}\right) \circ \operatorname{ad}_{g}=0$ and $-g \circ \operatorname{ad}_{\left(\langle x,\rangle \circ \mathbf{M}_{f}\right)}+\left[f \circ \mathbf{M}_{x}, g\right]_{2}=0$. But $\left(x \circ \mathbf{M}_{f}\right) \circ \operatorname{ad}_{g}(h)=$ $x\left(f \bullet_{2}[g, h]_{2}\right)=0$, for $h \in \mathfrak{Q}^{*}$, by hypothesis. Then $g \circ \mathrm{ad}_{\left(\langle x,\rangle \circ \mathbf{M}_{f}\right)}=\left[f \circ \mathbf{M}_{x}, g\right]_{2}$, getting condition (3.11). For $y \in \mathfrak{Q}$ we have

$[x \bullet f, y]=\left[x \circ \mathbf{M}_{f}+f \circ \mathbf{M}_{x}, y\right]=\left[x \circ \mathbf{M}_{f}, y\right]+\left[f \circ \mathbf{M}_{x}, y\right]=\left[x \circ \mathbf{M}_{f}, y\right]-y \circ \operatorname{ad}_{f \circ \mathbf{M}_{x}}+\left(f \circ \mathbf{M}_{x}\right) \circ \operatorname{ad}_{y}$.

then, using $[x \bullet f, y]=0$ we obtain $\left[x \circ \mathbf{M}_{f}, y\right]-y \circ \operatorname{ad}_{f \circ \mathbf{M}_{x}}=0$ and $\left(f \circ \mathrm{L}_{x}\right) \circ \operatorname{ad}_{y}=0$. But $\left(f \circ \mathbf{M}_{x}\right) \circ \operatorname{ad}_{y}(z)=$ $f\left(x \bullet_{1}[y, z]_{1}\right)=0$, for $z \in \mathfrak{L}$, by hypothesis. It remains condition $\left[x \circ \mathbf{M}_{f}, y\right]=y \circ \mathrm{ad}_{f \circ \mathbf{M}_{x}}$, which we prove that is equivalent to condition (3.11) already obtained. Indeed, for $g \in \mathfrak{L}^{*}, g\left(\left[x \circ \mathbf{M}_{f}, y\right]\right)=g \circ \operatorname{ad}_{x \circ \mathbf{M}_{f}}(y)$ and on the other hand

$$
g\left(y \circ \operatorname{ad}_{f \circ \mathbf{M}_{x}}\right)=\left(y \circ \operatorname{ad}_{f \circ \mathbf{M}_{x}}\right)(g)=y\left(\left[f \circ \mathbf{M}_{x}, g\right]\right)=\left[f \circ \mathbf{M}_{x}, g\right](y),
$$

as required.

Now we prove $(2) \Longrightarrow(3)$. Since $[f, g]_{2}\left(x \bullet_{1} y\right)=\left\langle[f, g]_{2}, x \bullet_{1} y\right\rangle=\left\langle f \otimes g, \Delta_{\mathbb{Q}}\left(x \bullet_{1} y\right)\right\rangle$, then from $[f, g]_{2}\left(x \bullet_{1} y\right)=0$ it turns out that $\Delta_{\mathfrak{L}}\left(x \bullet_{1} y\right)=0$ getting condition (3.12). As $\left(f \bullet_{2} g\right)[x, y]_{1}=\left\langle f \bullet_{2}\right.$ $\left.g,[x, y]_{1}\right\rangle=\left\langle f \otimes g, \Delta_{a}\left([x, y]_{1}\right)\right\rangle$, so from $\left(f \bullet_{2} g\right)[x, y]_{1}=0$ we conclude that $\Delta_{a}\left([x, y]_{1}\right)=0$ which implies condition (3.13).

$$
\begin{aligned}
\left\langle g \circ \operatorname{ad}_{\left(\left\langle x,>\circ \mathrm{M}_{f}, y\right\rangle\right.}\right. & =\left\langle g,\left[x \circ \mathrm{M}_{f}, y\right]_{1}\right\rangle=\left\langle\Delta_{\mathfrak{Q}}(g),\left(x \circ \mathrm{M}_{f}\right) \otimes y\right\rangle \\
& =\left\langle\sum_{(g)_{\mathfrak{I}}} g_{(1)} \otimes g_{(2)},\left(x \circ \mathrm{M}_{f}\right) \otimes y\right\rangle=\sum_{(g)_{\mathfrak{I}}}\left\langle g_{(1)}, x \circ \mathrm{M}_{f}\right\rangle\left\langle g_{(2)}, y\right\rangle \\
& =\sum_{(g)_{\mathfrak{I}}}\left\langle x, f \bullet_{2} g_{(1)}\right\rangle\left\langle y, g_{(2)}\right\rangle=\sum_{(g)_{\mathfrak{I}}}\left\langle\Delta_{a}(x), f \otimes g_{(1)}\right\rangle\left\langle y, g_{(2)}\right\rangle \\
& =\left\langle\Delta_{a}(x) \otimes y, \sum_{(g)_{\mathfrak{I}}} f \otimes g_{(1)} \otimes g_{(2)}\right\rangle=\left\langle\Delta_{a}(x) \otimes y, f \otimes \Delta_{\mathfrak{L}}(g)\right\rangle \\
& =\left\langle\Delta_{a}(x) \otimes y,\left(\mathrm{I} \otimes \Delta_{\mathfrak{L}}\right)(f \otimes g)\right\rangle=\left\langle\left(\mathrm{I} \otimes[,]_{1}\right)\left(\Delta_{a}(x) \otimes y\right), f \otimes g\right\rangle \\
& =\left\langle\sum_{(x)_{a}} x_{(1)} \otimes\left[x_{(2)}, y\right]_{1}, f \otimes g\right\rangle=\left\langle\Delta_{a}(x) \cdot \mathfrak{I} y, f \otimes g\right\rangle .
\end{aligned}
$$

On the other hand

$$
\begin{aligned}
\left\langle\left[f \circ \mathbf{M}_{x}, g\right], y\right\rangle & =\left\langle\left(f \circ \mathbf{M}_{x}\right) \otimes g, \Delta_{\mathfrak{L}}(y)\right\rangle=\left\langle\left(f \circ \mathbf{M}_{x}\right) \otimes g, \sum_{(y)_{\mathfrak{I}}} y_{(1)} \otimes y_{(2)}\right\rangle \\
& =\sum_{(y)_{\mathfrak{I}}}\left\langle f, x \bullet_{1} y_{(1)}\right\rangle\left\langle g, y_{(2)}\right\rangle=\left\langle f \otimes g, \sum_{(y)_{\mathfrak{L}}}\left(x \bullet_{1} y_{(1)}\right) \otimes y_{(2)}\right\rangle \\
& =\left\langle f \otimes g, x \cdot{ }_{a} \Delta_{\mathfrak{L}}(y)\right\rangle
\end{aligned}
$$


Therefore, from $g \circ \operatorname{ad}_{\left(\left\langle x,>\circ \mathbf{M}_{f}\right)\right.}=\left[f \circ \mathbf{M}_{x}, g\right]$ we conclude that $x \cdot{ }_{a} \Delta_{\mathfrak{L}}(y)-\Delta_{a}(x) \cdot \mathfrak{g} y=0$ as required. As in above proposition, we prove the desired equivalences.

Proposition 3.7. The following assertions are equivalent:

(1) $[\mathcal{D}(\mathfrak{L}), \mathcal{D}(\mathfrak{L})] \subseteq \operatorname{Ann}\left((\mathcal{D}(\mathfrak{L}))^{+}\right)$;

(2) For any $x, y \in \mathfrak{L}$ and $f, g \in \mathfrak{Q}^{*}$,

$$
\begin{aligned}
& {[f, g]_{2}\left(x \bullet \bullet_{1} y\right)=0,} \\
& (f \bullet 2 g)[x, y]_{1}=0, \\
& g \circ M_{\left(\left\langle x,>\circ a d_{f}\right)\right.}=\left(f \circ a d_{x}\right) \bullet_{2} g ;
\end{aligned}
$$

(3) For any $x, y \in \mathfrak{L}$,

$$
\begin{aligned}
& \Delta_{\mathfrak{L}}\left(x \bullet_{1} y\right)=0, \\
& \Delta_{a}\left([x, y]_{1}\right)=0, \\
& x \cdot \mathfrak{L} \Delta_{a}(y)-\Delta_{\mathfrak{Q}}(x) \cdot{ }_{a} y=0 .
\end{aligned}
$$

Proof. First we show (1) $\Longrightarrow(2)$. We use a similar reasoning to the one applied in Proposition 3.6. Again, we have to study three cases. First, we take $x, y \in \mathfrak{L}$. Since $[\mathfrak{L}, \mathfrak{L}] \subseteq \operatorname{Ann}\left(\mathfrak{L}^{+}\right)$then it is enough to take $f \in \mathfrak{Q}^{*}$. From $[x, y] \bullet f=0$ we infer $\left(f \bullet_{2} g\right)[x, y]_{1}=0$ already appeared in Proposition 3.6.

Next, we take $f, g \in \mathfrak{L}^{*}$. Since $\left[\mathfrak{Q}^{*}, \mathfrak{Q}^{*}\right] \subseteq \operatorname{Ann}\left(\left(\mathfrak{L}^{*}\right)^{+}\right)$then we just take $x \in \mathfrak{L}$. From $[f, g] \bullet x=0$ it follows $[f, g]_{2}(x \bullet 1, y)=0$ obtained also in Proposition 3.6.

Finally, we take $x \in \mathfrak{L}$ and $f \in \mathfrak{Q}^{*}$. For $g \in \mathfrak{L}^{*}$, we have

$$
[x, f] \bullet g=\left(x \circ \operatorname{ad}_{f}-f \circ \operatorname{ad}_{x}\right) \bullet g=\left(x \circ \operatorname{ad}_{f}\right) \circ \mathbf{M}_{g}+g \circ \mathbf{M}_{\left(\left\langle x,>\circ \operatorname{ad}_{f}\right)\right.}-\left(f \circ \operatorname{ad}_{x}\right) \bullet 2 g .
$$

Using the fact that $[x, f] \bullet g=0$ we obtain $\left(x \circ \operatorname{ad}_{f}\right) \circ \mathbf{M}_{g}=0$ and $g \circ \mathbf{M}_{\left(\langle x,\rangle \circ \mathrm{ad}_{f}\right)}-\left(f \circ \operatorname{ad}_{x}\right) \bullet 2 g=0$. But $\left(x \circ \operatorname{ad}_{f}\right) \circ \mathbf{M}_{g}(h)=x\left([f, g \bullet 2 h]_{2}\right)=0$, for $h \in \mathfrak{L}^{*}$, by hypothesis. Thus $g \circ \mathbf{M}_{\left(\left\langle x,>\circ d_{f}\right)\right.}=\left(f \circ \operatorname{ad}_{x}\right) \bullet 2 g$, getting condition (3.15). Given $y \in \mathfrak{L}$, we obtain

$$
[x, f] \bullet y=\left(x \circ \operatorname{ad}_{f}-f \circ \operatorname{ad}_{x}\right) \bullet y=\left(x \circ \operatorname{ad}_{f}\right) \bullet 1 y-y \circ \mathbf{M}_{f \circ a_{x}}-\left(f \circ \operatorname{ad}_{x}\right) \circ \mathbf{M}_{y}
$$

from $[x, f] \bullet y=0$ we have $\left(x \circ \operatorname{ad}_{f}\right) \bullet 1, y-y \circ \mathbf{M}_{f \circ a_{x}}=0$ and $\left(f \circ \operatorname{ad}_{x}\right) \circ \mathbf{M}_{y}=0 . \operatorname{But}\left(f \circ \operatorname{ad}_{x}\right) \circ \mathbf{M}_{y}(z)=$ $f\left(\left[x, y \bullet \bullet_{1} z\right]_{1}\right)=0$, for $z \in \mathfrak{L}$, by hypothesis. It remains condition $\left(x \circ \operatorname{ad}_{f}\right) \bullet \bullet_{1} y=y \circ \mathbf{M}_{f \circ d_{x}}$, which we prove that is equivalent to condition (3.15) already obtained. Indeed, for $g \in \mathfrak{L}^{*}, g\left(\left(x \circ \operatorname{ad}_{f}\right) \bullet \bullet_{1} y\right)=$ $\left(g \circ \mathbf{M}_{\left(\langle x,\rangle \circ \mathrm{ad}_{f}\right)}\right)(y)$ and on the other hand

$$
g\left(y \circ \mathbf{M}_{f \circ \mathrm{ad}_{x}}\right)=\left(y \circ \mathbf{M}_{f \circ \mathrm{ad}_{x}}\right)(g)=y\left(\left(f \circ \mathrm{ad}_{x}\right) \bullet 2 g\right)=\left(\left(f \circ \operatorname{ad}_{x}\right) \bullet g\right)(y),
$$

as required.

Now we prove (2) $\Longrightarrow$ (3). We just need to study the last statement. Given $x, y \in \mathfrak{L}, f, g \in \mathfrak{L}^{*}$, we get

$$
\begin{aligned}
\left\langle g \circ \mathrm{M}_{\left(\langle x,\rangle \circ \mathrm{ad}_{f}\right)}, y\right\rangle & =\left\langle g,\left(x \circ \operatorname{ad}_{f}\right) \bullet 1, y\right\rangle=\left\langle\Delta_{a}(g),\left(x \circ \operatorname{ad}_{f}\right) \otimes y\right\rangle \\
& =\left\langle\sum_{(g)_{a}} g_{(1)} \otimes g_{(2)},\left(x \circ \operatorname{ad}_{f}\right) \otimes y\right\rangle=\sum_{(g)_{a}}\left\langle g_{(1)}, x \circ \operatorname{ad}_{f}\right\rangle\left\langle g_{(2)}, y\right\rangle \\
& =\sum_{()_{a}}\left\langle x,\left[f, g_{(1)}\right]_{2}\right\rangle\left\langle y, g_{(2)}\right\rangle=\sum_{(g)_{a}}\left\langle\Delta_{\mathfrak{L}}(x), f \otimes g_{(1)}\right\rangle\left\langle y, g_{(2)}\right\rangle \\
& =\left\langle\Delta_{\mathfrak{L}}(x) \otimes y, \sum_{(g)_{a}} f \otimes g_{(1)} \otimes g_{(2)}\right\rangle=\left\langle\Delta_{\mathfrak{L}}(x) \otimes y, f \otimes \Delta_{a}(g)\right\rangle \\
& =\left\langle\Delta_{\mathfrak{L}}(x) \otimes y,\left(\mathrm{I} \otimes \Delta_{a}\right)(f \otimes g)\right\rangle=\left\langle\left(\mathrm{I} \otimes \bullet_{1}\right)\left(\Delta_{\mathfrak{L}}(x) \otimes y\right), f \otimes g\right\rangle \\
& =\left\langle\sum_{(x)_{\mathfrak{L}}} x_{(1)} \otimes\left(x_{(2)} \bullet y\right), f \otimes g\right\rangle=\left\langle\Delta_{\mathfrak{L}}(x) \cdot a y, f \otimes g\right\rangle .
\end{aligned}
$$


On the other hand

$$
\begin{aligned}
\left\langle\left(f \circ \mathrm{ad}_{x}\right) \bullet g, y\right\rangle & =\left\langle\left(f \circ \mathrm{ad}_{x}\right) \otimes g, \Delta_{a}(y)\right\rangle=\left\langle\left(f \circ \mathrm{ad}_{x}\right) \otimes g, \sum_{(y)_{a}} y_{(1)} \otimes y_{(2)}\right\rangle \\
& =\sum_{(y)_{a}}\left\langle f, x \bullet \bullet_{1} y_{(1)}\right\rangle\left\langle g, y_{(2)}\right\rangle=\left\langle f \otimes g, \sum_{(y)_{a}}\left(\left[x, y_{(1)}\right]_{1}\right) \otimes y_{(2)}\right\rangle \\
& =\left\langle f \otimes g, x \cdot \bullet_{\mathfrak{I}} \Delta_{a}(y)\right\rangle
\end{aligned}
$$

Therefore, from $g \circ \mathbf{M}_{\left(\left\langle x,>\operatorname{ad}_{f}\right)\right.}=\left(f \circ \operatorname{ad}_{x}\right) \bullet g$ we conclude that $x \cdot \mathfrak{\Omega} \Delta_{a}(y)-\Delta_{\mathfrak{Q}}(x) \cdot_{a} y=0$ as desired. We complete the proof as in the previous propositions.

Now we summarize the results above in the following theorem.

Theorem 3.8. Let $(\mathfrak{L},$.$) be a symmetric Leibniz algebra and \Delta$ a coproduct on $\mathfrak{L}$. The triple $(\mathfrak{L}, . ., \Delta)$ is a symmetric Leibniz bialgebra if and only if these conditions are satisfied:

(1) $\left(\mathfrak{R}, \Delta_{\mathfrak{L}}\right)$ is a Lie coalgebra,

(2) $\left(\Delta_{a} \otimes I\right) \circ \Delta_{a}=\left(\Delta_{a} \otimes I\right) \circ \Delta_{\mathfrak{l}}=\left(\Delta_{\mathfrak{L}} \otimes I\right) \circ \Delta_{a}=0$,

(3) $\Delta_{a}\left(x \bullet_{1} y\right)=\Delta_{\mathfrak{I}}\left(x \bullet_{1} y\right)=\Delta_{a}\left([x, y]_{1}\right)=0$,

(4) $y \cdot{ }_{a} \Delta_{a}(x)+\Delta_{a}(y) \cdot{ }_{a} x=0$,

(5) $x \cdot{ }_{a} \Delta_{\mathfrak{L}}(y)-\Delta_{a}(x) \cdot \mathfrak{z} y=0$,

(6) $x \cdot \mathfrak{L} \Delta_{a}(y)-\Delta_{\mathfrak{L}}(x) \cdot{ }_{a} y=0$,

for any $x, y \in \mathfrak{Q}$.

\section{Coboundary Leibniz bialgebras, the Yang-BaXter equation and $r$-Matrices}

In the previous section we considered Leibniz bialgebras in general, now we are going to study a special class of them, namely the coboundary Leibniz bialgebras. It will give us the natural framework to study the analogue of the Yang-Baxter equation and r-matrix in our setting. Similar to the preceding section, first we present a brief description of the theory for Lie bialgebras as well as associative bialgebras.

A Lie bialgebra $\left(\mathfrak{g},[],, \Delta_{\mathfrak{L}}\right)$ is called coboundary if $\Delta_{\mathfrak{L}} \in \mathrm{B}^{1}\left(\mathfrak{g}, \mathfrak{g} \otimes \mathfrak{g}\right.$ ) (meaning that, $\Delta_{\mathfrak{L}}$ is a coboundary of $\mathfrak{g}$ with values in $\mathfrak{g} \otimes \mathfrak{g})$. In this case, there exists $r \in \mathfrak{g} \otimes \mathfrak{g}$ such that:

$$
\Delta_{\mathfrak{L}}(x)=\left(\operatorname{ad}_{x} \otimes \mathrm{I}+\mathrm{I} \otimes \operatorname{ad}_{x}\right)(r), \quad \forall x \in \mathfrak{g} .
$$

If $r=\sum_{i=1}^{n} a_{i} \otimes b_{i}$ with $a_{i}, b_{i} \in \mathfrak{g}, \forall i \in\{1, \ldots, n\}$, we consider $r_{12}=\sum_{i=1}^{n} a_{i} \otimes b_{i} \otimes 1, r_{13}=\sum_{i=1}^{n} a_{i} \otimes 1 \otimes b_{i}$ and $r_{23}=\sum_{i=1}^{n} 1 \otimes a_{i} \otimes b_{i}$ in $\mathfrak{U}(\mathfrak{g})^{\otimes^{3}}$, then

$$
\begin{aligned}
& {\left[r_{12}, r_{13}\right]=\sum_{i, j=1}^{n}\left[a_{i}, a_{j}\right] \otimes b_{i} \otimes b_{j},} \\
& {\left[r_{12}, r_{23}\right]=\sum_{i, j=1}^{n} a_{i} \otimes\left[b_{i}, a_{j}\right] \otimes b_{j},} \\
& {\left[r_{13}, r_{23}\right]=\sum_{i, j=1}^{n} a_{i} \otimes a_{j} \otimes\left[b_{i}, b_{j}\right],} \\
& C(r):=\left[r_{12}, r_{13}\right]+\left[r_{12}, r_{23}\right]+\left[r_{13}, r_{23}\right] .
\end{aligned}
$$

Proposition 4.1. [11] Let $\mathfrak{g}$ be a Lie algebra and $r \in \mathfrak{g} \otimes \mathfrak{g}$. The map $\Delta_{\mathfrak{L}}$ given by

$$
\Delta_{\mathfrak{Q}}(x)=\left(a d_{x} \otimes I+I \otimes a d_{x}\right)(r), \quad \forall x \in \mathfrak{g},
$$

defines a Lie bialgebra structure on $\mathfrak{g}$ if and only if the following statements are satisfied:

(1) $\left(a d_{x} \otimes I+I \otimes a d_{x}\right)(r+\tau(r))=0, \forall x \in \mathfrak{g} ;$ 
(2) $\left(a d_{x} \otimes I \otimes I+I \otimes a d_{x} \otimes I+I \otimes I \otimes a d_{x}\right) C(r)=0, \forall x \in \mathfrak{g}$.

The equation $C(r)=0$ is called classical Yang-Baxter equation (CYBE). If $r \in \mathfrak{g} \otimes \mathfrak{g}$ satisfies $C(r)=0$, then $r$ is called the solution of CYBE (as well a $r$-matrix of $\mathrm{g}$ ). It is clear that if $r$ is skew-symmetric (recall that $r \in \mathfrak{L} \otimes \mathfrak{L}$ is skew-symmetric if $r+\tau(r)=0)$ and $C(r)=0$, then $\left(\mathfrak{g},[],, \Delta_{\mathfrak{l}}\right)$ is a Lie bialgebra, where $\Delta_{\mathfrak{Q}}$ is the coalgebra structure defined by $r$. In this case, $\left(\mathfrak{g},[],, \Delta_{\mathfrak{L}}\right)$ is said a triangular Lie bialgebra. If $\left(\mathfrak{g},[],, \Delta_{\mathfrak{l}}\right.$ ) (with $\Delta_{\mathfrak{l}}(x)=x \cdot r, x \in \mathfrak{g}$ ) is a Lie bialgebra and $r$ a solution of CYBE which is not skew-symmetric, then $\left(\mathfrak{g},[],, \Delta_{\mathfrak{l}}\right)$ is called quasi-triangular Lie bialgebra and $r$ is called a quasi-classical $r$-matrix of $\mathfrak{g}$.

Now, let $(A, \bullet)$ be an associative algebra. If $r=\sum_{i=1}^{n} a_{i} \otimes b_{i} \in A \otimes A$, we consider

$$
\begin{aligned}
& r_{13} r_{12}=\sum_{i, j=1}^{n}\left(a_{i} \bullet a_{j}\right) \otimes b_{j} \otimes b_{i}, \\
& r_{12} r_{23}=\sum_{i, j=1}^{n} a_{i} \otimes\left(b_{i} \bullet a_{j}\right) \otimes b_{j}, \\
& r_{23} r_{13}=\sum_{i, j=1}^{n} a_{j} \otimes a_{i} \otimes\left(b_{i} \bullet b_{j}\right), \\
& A(r):=r_{13} r_{12}-r_{12} r_{23}+r_{23} r_{13} .
\end{aligned}
$$

Now, we define the coproduct $\Delta_{a}: A \longrightarrow A \otimes A$ by:

$$
\Delta_{a}(x)=\left(\mathrm{M}_{x} \otimes \mathrm{I}-\mathrm{I} \otimes \mathrm{M}_{x}\right)(r)=\sum_{i=1}^{n}\left(\left(x \bullet a_{i}\right) \otimes b_{i}-a_{i} \otimes\left(b_{i} \bullet x\right)\right), \quad \forall x \in A .
$$

It is easy to verify that the associativity of $\bullet$ implies condition (3.3).

Proposition 4.2. [1] $\left(A, \bullet, \Delta_{a}\right)$, where $\Delta_{a}$ is defined by $r \in A \otimes A$, is coassociative if and only if

$$
(L(x) \otimes I \otimes I-I \otimes I \otimes R(x)) A(r)=0, \quad \forall x \in A .
$$

Corollary 4.3. [2] The triple $\left(A, \bullet, \Delta_{a}\right)$, where as before $\Delta_{a}$ is given by $r \in A \otimes A$, is an infinitesimal bialgebra if and only if

$$
(L(x) \otimes I \otimes I-I \otimes I \otimes R(x)) A(r)=0, \quad \forall x \in A .
$$

In this case, $\left(A, \bullet, \Delta_{a}\right)$ is called a coboundary infinitesimal bialgebra.

The equation $A(r)=0$ is called the associative Yang-Baxter equation (AYBE), and each solution $r$ of this equation is called a solution of AYBE. We recall that a coalgebra $(A, \Delta)$ is called cocommutative if $\tau \circ \Delta=\Delta$

Definition 4.4. An infinitesimal bialgebra $\left(A, \bullet, \Delta_{a}\right)$ is called commutative if $(A, \bullet)$ is a commutative algebra and $\left(A, \Delta_{a}\right)$ is a cocommutative coalgebra.

It is clear that if $(A, \bullet)$ is a commutative associative algebra and $\Delta_{a}$ a coproduct on $A$ defined by $r \in A \otimes A$, the triple $\left(A, \bullet, \Delta_{a}\right)$ is a commutative infinitesimal bialgebra if and only if

(i) $(L(x) \otimes \mathrm{I}-\mathrm{I} \otimes L(x))(r+\tau(r))=0$;

(ii) $(L(x) \otimes \mathrm{I} \otimes \mathrm{I}-\mathrm{I} \otimes \mathrm{I} \otimes L(x)) A(r)=0$,

hold $\forall x \in A$.

Now, our goal is to transfer these structures to the Leibniz algebras. 
Definition 4.5. A Leibniz bialgebra $(\mathfrak{L}, ., \Delta)$ is called coboundary if there exists $r \in \mathfrak{L} \otimes \mathfrak{L}$ such that:

$$
\Delta(x)=\left(L_{x} \otimes \mathrm{I}-\mathrm{I} \otimes R_{x}\right)(r), \quad \forall x \in \mathfrak{L} .
$$

If $r$ is skew-symmetric, we obtain for any $x \in \mathfrak{R}$ :

$$
\begin{aligned}
& \Delta_{\mathfrak{Q}}(x)=\left(\operatorname{ad}_{x} \otimes \mathrm{I}+\mathrm{I} \otimes \mathrm{ad}_{x}\right)(r), \\
& \Delta_{a}(x)=\left(\mathrm{M}_{x} \otimes \mathrm{I}-\mathrm{I} \otimes \mathrm{M}_{x}\right)(r),
\end{aligned}
$$

thus $\left(\mathfrak{L},[,]_{1}, \Delta_{\mathfrak{L}}\right)\left(\right.$ resp. $\left.\left(\mathfrak{L}, \bullet_{1}, \Delta_{a}\right)\right)$ is actually a coboundary Lie bialgebra (resp. coboundary commutative associative bialgebra).

Remark 4.6. If $r \in \mathfrak{L} \otimes \mathfrak{Q}$ such that $(x \in \mathfrak{Q})$ :

$$
\begin{aligned}
& \Delta_{\mathfrak{Q}}(x)=\left(\operatorname{ad}_{x} \otimes \mathrm{I}+\mathrm{I} \otimes \mathrm{ad}_{x}\right)(r), \\
& \Delta_{a}(x)=\left(\mathrm{M}_{x} \otimes \mathrm{I}-\mathrm{I} \otimes \mathrm{M}_{x}\right)(r),
\end{aligned}
$$

then $\Delta(x)=\left(L_{x} \otimes \mathrm{I}-\mathrm{I} \otimes R_{x}\right)(r)$ holds for any $x \in \mathfrak{L}$, meaning that $(\mathfrak{L}, ., \Delta)$ is a coboundary Leibniz bialgebra.

Theorem 4.7. Let $(\mathfrak{L}$, , ) be a symmetric Leibniz algebra and $r \in \mathfrak{L} \otimes \mathfrak{L}$ a skew-symmetric element. We consider the coproduct $\Delta: \mathfrak{L} \longrightarrow \mathfrak{L} \otimes \mathfrak{L}$ defined by:

$$
\Delta(x)=\left(L_{x} \otimes I-I \otimes R_{x}\right)(r), \quad \forall x \in \mathfrak{R} .
$$

The triple $(\mathscr{L}, ., \Delta)$ is a Leibniz bialgebra if and only if these conditions are satisfied:

(1) $\left(a d_{x} \otimes I \otimes I+I \otimes a d_{x} \otimes I+I \otimes I \otimes a d_{x}\right) C(r)=0$,

(2) $\left(I \otimes I \otimes M_{x}\right) C(r)=0$,

(3) $\left(I \otimes I \otimes M_{x}\right) A(r)=0$,

(4) $\left(I \otimes I \otimes a d_{x}\right) A(r)=0$,

(5) $\left(M_{x} \otimes a d_{y}\right)(r)=0$,

for all $x, y \in \mathfrak{R}$.

Proof. Set $r=\sum_{i=1}^{n} a_{i} \otimes b_{i}-b_{i} \otimes a_{i}$, with $a_{i}, b_{i} \in \mathfrak{L}$ for all $i \in\{1, \ldots, n\}$. We have (for $x \in \mathfrak{L}$ ):

$$
\begin{aligned}
& \Delta_{\mathfrak{Q}}(x)=\left(\operatorname{ad}_{x} \otimes I+I \otimes \mathrm{ad}_{x}\right)(r)=\sum_{i=1}^{n}\left(\left[x, a_{i}\right]_{1} \otimes b_{i}-\left[x, b_{i}\right]_{1} \otimes a_{i}+a_{i} \otimes\left[x, b_{i}\right]_{1}-b_{i} \otimes\left[x, a_{i}\right]_{1}\right), \\
& \Delta_{a}(x)=\left(\mathrm{M}_{x} \otimes \mathrm{I}-\mathrm{I} \otimes \mathrm{M}_{x}\right)(r)=\sum_{i=1}^{n}\left(\left(x \bullet_{1} a_{i}\right) \otimes b_{i}-\left(x \bullet b_{1}\right) \otimes a_{i}-a_{i} \otimes\left(x \bullet b_{1} b_{i}\right)+b_{i} \otimes\left(x \bullet_{1} a_{i}\right)\right) .
\end{aligned}
$$

Since $r$ is skew-symmetric, $\left(\mathfrak{L}, \Delta_{\mathfrak{l}}\right)$ is a Lie coalgebra if and only if $\left(\operatorname{ad}_{x} \otimes \mathrm{I} \otimes \mathrm{I}+\mathrm{I} \otimes \mathrm{ad}_{x} \otimes \mathrm{I}+\mathrm{I} \otimes \mathrm{I} \otimes \mathrm{ad}_{x}\right) C(r)=0$. By easy calculation we prove that: $\forall x, y \in \mathfrak{L}$,

(i) $\left(\Delta_{a} \otimes \mathrm{I}\right) \circ \Delta_{a}(x)=0$ if and only if $\left(\mathrm{I} \otimes \mathrm{I} \otimes \mathrm{M}_{x}\right)\left(r_{13} r_{12}-r_{12} r_{23}\right)=0$ which is equivalent to $\left(\mathrm{I} \otimes \mathrm{I} \otimes \mathrm{M}_{x}\right) A(r)=0$, because $\left(\mathfrak{Q}^{+}\right)^{3}=\{0\}$.

(ii) $\left(\Delta_{a} \otimes \mathrm{I}\right) \circ \Delta_{\mathfrak{Q}}(x)=0$ if and only if $\left(\mathrm{I} \otimes \mathrm{I} \otimes \mathrm{ad}_{x}\right)\left(r_{13} r_{12}-r_{12} r_{23}\right)=0$ and equivalently $\left(\mathrm{I} \otimes \mathrm{I} \otimes \mathrm{ad}_{x}\right) A(r)=$ 0 , as $\left(\mathfrak{L}^{+}\right)^{2} \subseteq 3\left(\mathfrak{L}^{-}\right)$.

(iii) $\left(\Delta_{\mathfrak{I}} \otimes \mathrm{I}\right) \circ \Delta_{a}(x)=0$ if and only if $\left(\mathrm{I} \otimes \mathrm{I} \otimes \mathrm{M}_{x}\right)\left(\left[r_{12}, r_{13}\right]+\left[r_{12}, r_{23}\right]\right)=0$ which is the same as $\left(\mathrm{I} \otimes \mathrm{I} \otimes \mathrm{M}_{x}\right) C(r)=0$, since $\left[\mathfrak{L}^{-}, \mathfrak{L}^{-}\right]_{1} \subseteq \operatorname{Ann}\left(\mathfrak{L}^{+}\right)$.

(iv)

$$
\begin{aligned}
y \cdot{ }_{a} \Delta_{a}(x)+\Delta_{a}(y) \cdot{ }_{a} x & =\sum_{i=1}^{n}\left(-\left(y \bullet \bullet_{1} a_{i}\right) \otimes\left(x \bullet \bullet_{1} b_{i}\right)+\left(y \bullet{ }_{1} b_{i}\right) \otimes\left(x \bullet \bullet_{1} a_{i}\right)\right) \\
& +\sum_{i=1}^{n}\left(\left(y \bullet{ }_{1} a_{i}\right) \otimes\left(b_{i} \bullet \bullet_{1} x\right)-\left(y \bullet b_{i}\right) \otimes\left(a_{i} \bullet \bullet_{1} x\right)\right)=0
\end{aligned}
$$


(v) $x \cdot{ }_{a} \Delta_{\mathfrak{L}}(y)-\Delta_{a}(x) \cdot \mathfrak{L} y=0$ if and only if $\left(\mathrm{M}_{x} \otimes \mathrm{ad}_{y}\right)(r)=0$.

(vi) $x \cdot{ }_{\mathfrak{Q}} \Delta_{a}(y)-\Delta_{\mathfrak{Q}}(x) \cdot a y=0$ if and only if $\left(\operatorname{ad}_{x} \otimes \mathrm{M}_{y}\right)(r)=0$. The fact that $r$ is skew-symmetric implies that (v) and (vi) are equivalent.

(vii) Since $\left(\mathfrak{L}^{+}\right)^{3}=\{0\},\left(\mathfrak{L}^{+}\right)^{2} \subseteq z\left(\mathfrak{L}^{-}\right)$and $\left[\mathfrak{L}^{-}, \mathfrak{L}^{-}\right]_{1} \subseteq \operatorname{Ann}\left(\mathfrak{L}^{+}\right)$, then $\Delta_{a}\left(x \bullet_{1} y\right)=0, \Delta_{\mathfrak{L}}\left(x \bullet \bullet_{1} y\right)=0$ and $\Delta_{a}\left([x, y]_{1}\right)=0$.

By Theorem 3.8, we conclude that $(\mathfrak{L}, ., \Delta)$ is a Leibniz bialgebra if and only if (i)-(vii) are satisfied.

We have the following immediate consequences:

Corollary 4.8. If $C(r)=0,(\mathfrak{L}, ., \Delta)$ is a Leibniz bialgebra if and only if these assertions are satisfied:

(1) $\left(I \otimes I \otimes M_{x}\right) A(r)=0$,

(2) $\left(I \otimes I \otimes a d_{x}\right) A(r)=0$,

(3) $\left(M_{x} \otimes a d_{y}\right)(r)=0$,

for $x, y \in \mathfrak{L}$.

Corollary 4.9. If $A(r)=0,(\mathfrak{L}, ., \Delta)$ is a Leibniz bialgebra if and only if these conditions are satisfied:

(1) $\left(a d_{x} \otimes I \otimes I+I \otimes a d_{x} \otimes I+I \otimes I \otimes a d_{x}\right) C(r)=0$,

(2) $\left(I \otimes I \otimes M_{x}\right) C(r)=0$,

(3) $\left(M_{x} \otimes a d_{y}\right)(r)=0$,

for $x, y \in \mathfrak{L}$.

Corollary 4.10. If $C(r)=A(r)=0,(\mathfrak{Q}, ., \Delta)$ is a Leibniz bialgebra if and only if $\left(M_{x} \otimes a d_{y}\right)(r)=0$, for $x, y \in \mathfrak{R}$.

The following proposition presents an interesting construction of a Leibniz bialgebra, which is not a coboundary Leibniz bialgebra.

Proposition 4.11. Consider $(\mathfrak{L},$.$) a symmetric Leibniz algebra together with an element r=\sum_{i=1}^{n}\left(a_{i} \otimes\right.$ $\left.b_{i}-b_{i} \otimes a_{i}\right)$ in $\mathfrak{L} \otimes \mathfrak{L}$. Define the following maps $\Delta_{\mathfrak{L}}, \Delta_{a}: \mathfrak{L} \longrightarrow \mathfrak{L} \otimes \mathfrak{L}$ by (for any $\left.x \in \mathfrak{L}\right)$ :

$$
\begin{aligned}
& \Delta_{\mathfrak{L}}(x)=\left(a d_{x} \otimes I+I \otimes a d_{x}\right)(r), \\
& \Delta_{a}(x)=\left(-M_{x} \otimes I+I \otimes M_{x}\right)(r) .
\end{aligned}
$$

Then $\mathfrak{Q}$ is a Leibniz bialgebra with the coproducts $\Delta_{\mathfrak{I}}$ and $\Delta_{a}$ on $\mathfrak{Q}\left(\right.$ hence $\Delta(x)=\left(-R_{x} \otimes I+I \otimes L_{x}\right)(r)$, for any $x \in \mathfrak{L})$ if and only if the following condition is true:

$$
\left(a d_{x} \otimes I \otimes I+I \otimes a d_{x} \otimes I+I \otimes I \otimes a d_{x}\right) C(r)=0,
$$

for any $x \in \mathfrak{Q}$.

Explicitly, $\Delta_{\mathfrak{L}}$ and $\Delta_{a}$ can be written as (for any $x \in \mathfrak{L}$ ):

$$
\begin{aligned}
& \Delta_{\mathfrak{Q}}(x)=\left(\operatorname{ad}_{x} \otimes I+I \otimes \operatorname{ad}_{x}\right)(r)=\sum_{i=1}^{n}\left(\left[x, a_{i}\right]_{1} \otimes b_{i}-\left[x, b_{i}\right]_{1} \otimes a_{i}+a_{i} \otimes\left[x, b_{i}\right]_{1}-b_{i} \otimes\left[x, a_{i}\right]_{1}\right), \\
& \Delta_{a}(x)=\left(-\mathrm{M}_{x} \otimes \mathrm{I}+\mathrm{I} \otimes \mathrm{M}_{x}\right)(r)=\sum_{i=1}^{n}\left(-\left(x \bullet_{1} a_{i}\right) \otimes b_{i}+\left(x \bullet_{1} b_{i}\right) \otimes a_{i}+a_{i} \otimes\left(x \bullet \bullet_{1} b_{i}\right)-b_{i} \otimes\left(x \bullet_{1} a_{i}\right)\right) .
\end{aligned}
$$

Proof. Straightforward calculations show that the conditions of Theorem 3.8 are checked. Indeed, conditions (2) and (3) are immediate. $\left(\mathfrak{L}, \Delta_{\mathfrak{L}}\right)$ is a Lie coalgebra, means that, $\operatorname{Im}\left(\Delta_{\mathfrak{L}}\right) \subseteq \operatorname{Im}(\mathrm{I}-\tau)$ and 
$\left(\mathrm{I}+\xi+\xi^{2}\right) \circ\left(\mathrm{I} \otimes \Delta_{\mathfrak{L}}\right) \circ \Delta_{\mathfrak{L}}=0$. Doing some careful computations we obtain (with $\left.x \in \mathfrak{L}\right)$ :

$\left(\mathrm{I} \otimes \Delta_{\mathfrak{L}}\right) \circ \Delta_{\mathfrak{L}}(x)=\left(\mathrm{I} \otimes \Delta_{\mathfrak{L}}\right)\left(\sum_{i=1}^{n}\left(\left[x, a_{i}\right]_{1} \otimes b_{i}-\left[x, b_{i}\right]_{1} \otimes a_{i}+a_{i} \otimes\left[x, b_{i}\right]_{1}-b_{i} \otimes\left[x, a_{i}\right]_{1}\right)\right)$

$=\sum_{i=1}^{n}\left(\left[x, a_{i}\right]_{1} \otimes \Delta_{\mathfrak{L}}\left(b_{i}\right)-\left[x, b_{i}\right]_{1} \otimes \Delta_{\mathfrak{L}}\left(a_{i}\right)+a_{i} \otimes \Delta_{\mathfrak{L}}\left(\left[x, b_{i}\right]_{1}\right)-b_{i} \otimes \Delta_{\mathfrak{L}}\left(\left[x, a_{i}\right]_{1}\right)\right)$

$=\sum_{i, j=1}^{n}\left(\left[x, a_{i}\right]_{1} \otimes\left[b_{i}, a_{j}\right]_{1} \otimes b_{j}-\left[x, a_{i}\right]_{1} \otimes\left[b_{i}, b_{j}\right]_{1} \otimes a_{j}+\left[x, a_{i}\right]_{1} \otimes a_{j} \otimes\left[b_{i}, b_{j}\right]_{1}-\left[x, a_{i}\right]_{1} \otimes b_{j} \otimes\left[b_{i}, a_{j}\right]_{1}\right.$

$-\left[x, b_{i}\right]_{1} \otimes\left[a_{i}, a_{j}\right]_{1} \otimes b_{j}+\left[x, b_{i}\right]_{1} \otimes\left[a_{i}, b_{j}\right]_{1} \otimes a_{j}-\left[x, b_{i}\right]_{1} \otimes a_{j} \otimes\left[a_{i}, b_{j}\right]_{1}+\left[x, b_{i}\right]_{1} \otimes b_{j} \otimes\left[a_{i}, a_{j}\right]_{1}$

$+a_{i} \otimes\left[\left[x, b_{i}\right]_{1}, a_{j}\right]_{1} \otimes b_{j}-a_{i} \otimes\left[\left[x, b_{i}\right]_{1}, b_{j}\right]_{1} \otimes a_{j}+a_{i} \otimes a_{j} \otimes\left[\left[x, b_{i}\right]_{1}, b_{j}\right]_{1}-a_{i} \otimes b_{j} \otimes\left[\left[x, b_{i}\right]_{1}, a_{j}\right]_{1}$

$\left.-b_{i} \otimes\left[\left[x, a_{i}\right]_{1}, a_{j}\right]_{1} \otimes b_{j}+b_{i} \otimes\left[\left[x, a_{i}\right]_{1}, b_{j}\right]_{1} \otimes a_{j}-b_{i} \otimes a_{j} \otimes\left[\left[x, a_{i}\right]_{1}, b_{j}\right]_{1}+b_{i} \otimes b_{j} \otimes\left[\left[x, a_{i}\right]_{1}, a_{j}\right]_{1}\right)$

and since $r$ is skew-symmetric we have,

$$
\left(\mathrm{I}+\xi+\xi^{2}\right) \circ\left(\mathrm{I} \otimes \Delta_{\mathfrak{L}}\right) \circ \Delta_{\mathfrak{L}}(x)=\left(\operatorname{ad}_{x} \otimes \mathrm{I} \otimes \mathrm{I}+\mathrm{I} \otimes \mathrm{ad}_{x} \otimes \mathrm{I}+\mathrm{I} \otimes \mathrm{I} \otimes \mathrm{ad}_{x}\right) C(r) .
$$

Thus, $\left(\mathfrak{L}, \Delta_{\mathfrak{L}}\right)$ is a Lie coalgebra if and only if condition (4.17) is verified. Next we take care about condition (4). Since $\left(\mathfrak{L}^{+}\right)^{3}=\{0\}$, we have (for $x, y \in \mathfrak{L}$ )

$$
\begin{aligned}
y \cdot{ }_{a} \Delta_{a}(x) & =\sum_{i=1}^{n}\left(-y \bullet_{1}\left(x \bullet{ }_{1} a_{i}\right) \otimes b_{i}+y \bullet \bullet_{1}\left(x \bullet_{1} b_{i}\right) \otimes a_{i}+\left(y \bullet \bullet_{1} a_{i}\right) \otimes\left(x \bullet_{1} b_{i}\right)-\left(y \bullet b_{i}\right) \otimes\left(x \bullet{ }_{1} a_{i}\right)\right) \\
& =\sum_{i=1}^{n}\left(\left(y \bullet a_{1} a_{i}\right) \otimes\left(x \bullet_{1} b_{i}\right)-\left(y \bullet_{1} b_{i}\right) \otimes\left(x \bullet \bullet_{1} a_{i}\right)\right),
\end{aligned}
$$

and

$$
\begin{aligned}
\Delta_{a}(y) \cdot{ }_{a} x & =\sum_{i=1}^{n}\left(-\left(y \bullet_{1} a_{i}\right) \otimes\left(b_{i} \bullet \bullet_{1} x\right)+\left(y \bullet_{1} b_{i}\right) \otimes\left(a_{i} \bullet 1 x\right)+a_{i} \otimes\left(y \bullet_{1} b_{i}\right) \bullet_{1} x-b_{i} \otimes\left(y \bullet \bullet_{1} a_{i}\right) \bullet \bullet_{1} x\right) \\
& =\sum_{i=1}^{n}\left(-\left(y \bullet_{1} a_{i}\right) \otimes\left(b_{i} \bullet_{1} x\right)+\left(y \bullet_{1} b_{i}\right) \otimes\left(a_{i} \bullet \bullet_{1} x\right)\right),
\end{aligned}
$$

which prove that $y \cdot{ }_{a} \Delta_{a}(x)+\Delta_{a}(y) \cdot{ }_{a} x=0$. Concerning condition $(5)$, since $\left(\mathfrak{L}^{+}\right)^{2} \subseteq z\left(\mathfrak{L}^{-}\right)$and $\left[\mathfrak{L}^{-}, \mathfrak{Q}^{-}\right] \subseteq$ $\operatorname{Ann}\left(\mathfrak{L}^{+}\right)$, we have

$$
\begin{aligned}
x \cdot{ }_{a} \Delta_{\mathfrak{Q}}(y) & =\sum_{i=1}^{n}\left(x \bullet_{1}\left[y, a_{i}\right]_{1} \otimes b_{i}-x \bullet_{1}\left[y, b_{i}\right]_{1} \otimes a_{i}+\left(x \bullet_{1} a_{i}\right) \otimes\left[y, b_{i}\right]_{1}-\left(x \bullet{ }_{1} b_{i}\right) \otimes\left[y, a_{i}\right]_{1}\right) \\
& =\sum_{i=1}^{n}\left(\left(x \bullet_{1} a_{i}\right) \otimes\left[y, b_{i}\right]_{1}-\left(x \bullet_{1} b_{i}\right) \otimes\left[y, a_{i}\right]_{1}\right)
\end{aligned}
$$

and

$$
\begin{aligned}
\Delta_{a}(x) \cdot \mathbb{Q} y & =\sum_{i=1}^{n}\left(-\left(x \bullet_{1} a_{i}\right) \otimes\left[b_{i}, y\right]_{1}+\left(x \bullet_{1} b_{i}\right) \otimes\left[a_{i}, y\right]_{1}+a_{i} \otimes\left[\left(x \bullet_{1} b_{i}\right), y\right]_{1}-b_{i} \otimes\left[\left(x \bullet \bullet_{1} a_{i}\right), y\right]_{1}\right) \\
& =\sum_{i=1}^{n}\left(\left(x \bullet{ }_{1} a_{i}\right) \otimes\left[y, b_{i}\right]_{1}-\left(x \bullet \bullet_{1} b_{i}\right) \otimes\left[y, a_{i}\right]_{1}\right)
\end{aligned}
$$


it follows $x \cdot{ }_{a} \Delta_{\mathfrak{L}}(y)-\Delta_{a}(x) \cdot \mathfrak{Q} y=0$. Finally, relative to condition (6), using $\left(\mathfrak{L}^{+}\right)^{2} \subseteq z\left(\mathfrak{I}^{-}\right)$and $\left[\mathfrak{I}^{-}, \mathfrak{L}^{-}\right] \subseteq$ $\operatorname{Ann}\left(\mathfrak{Q}^{+}\right)$, we have

$$
\begin{aligned}
x \cdot \mathfrak{Q} \Delta_{a}(y) & =\sum_{i=1}^{n}\left(-\left[x, y \bullet_{1} a_{i}\right]_{1} \otimes b_{i}+\left[x, y \bullet_{1} b_{i}\right]_{1} \otimes a_{i}+\left[x, a_{i}\right]_{1} \otimes\left(y \bullet_{1} b_{i}\right)-\left[x, b_{i}\right]_{1} \otimes\left(y \bullet{ }_{1} a_{i}\right)\right) \\
& =\sum_{i=1}^{n}\left(\left[x, a_{i}\right]_{1} \otimes\left(y \bullet \bullet_{1} b_{i}\right)-\left[x, b_{i}\right]_{1} \otimes\left(y \bullet_{1} a_{i}\right)\right)
\end{aligned}
$$

and

$$
\begin{aligned}
\Delta_{\mathfrak{L}}(x) \cdot_{a} y & =\sum_{i=1}^{n}\left(\left[x, a_{i}\right]_{1} \otimes\left(b_{i} \bullet_{1} y\right)-\left[x, b_{i}\right]_{1} \otimes\left(a_{i} \bullet_{1} y\right)+a_{i} \otimes\left[x, b_{i}\right]_{1} \bullet_{1} y-b_{i} \otimes\left[x, a_{i}\right]_{1} \bullet_{1} y\right) \\
& =\sum_{i=1}^{n}\left(\left[x, a_{i}\right]_{1} \otimes\left(y \bullet_{1} b_{i}\right)-\left[x, b_{i}\right]_{1} \otimes\left(y \bullet_{1} a_{i}\right)\right)
\end{aligned}
$$

obtaining $x \cdot \mathfrak{L} \Delta_{a}(y)-\Delta_{\mathfrak{L}}(x) \cdot{ }_{a} y=0$, completing the proof.

\section{Existence OF COBOUNDARY LEIBNIZ BIALGEBRA STRUCTURE ON A SYMMETRIC LEIBNIZ ALGEBRA}

Let $(\mathfrak{L},$.$) be a symmetric Leibniz algebra. We are interested in the case when \left(\mathfrak{L}^{+}\right)^{2} \neq\{0\}$ and $\left[\mathfrak{Q}^{-}, \mathfrak{Q}^{-}\right]_{1} \neq\{0\}$, i.e., when $(\mathfrak{L},$.$) is neither a Lie algebra nor an associative commutative algebra.$

Theorem 5.1. Let $(\mathfrak{Q},$.$) be a symmetric Leibniz algebra such that \left(\mathfrak{L}^{+}\right)^{2} \backslash\left[\mathfrak{L}^{-}, \mathfrak{L}^{-}\right]_{1} \neq\{0\}$. Then there exists a coboundary Leibniz bialgebra structure on $(\mathfrak{Q},$.$) such that \Delta_{\mathfrak{L}} \neq 0$ and $\Delta_{a} \neq 0$.

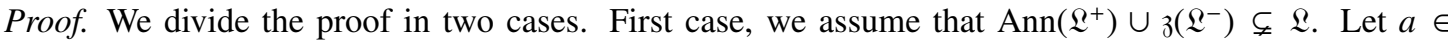
$\mathfrak{L} \backslash\left(\operatorname{Ann}\left(\mathfrak{L}^{+}\right) \cup z\left(\mathfrak{L}^{-}\right)\right), b \in\left(\mathfrak{L}^{+}\right)^{2} \backslash\left[\mathfrak{L}^{-}, \mathfrak{L}^{-}\right]_{1}$ and we set $r:=a \otimes b-b \otimes a$. We have (for $\left.x \in \mathfrak{L}\right)$ :

$$
\begin{aligned}
\Delta(x) & =(x . a) \otimes b-(x . b) \otimes a-a \otimes(b . x)+b \otimes(a . x), \\
\Delta_{\mathfrak{L}}(x) & =[x, a]_{1} \otimes b-[x, b]_{1} \otimes a+a \otimes[x, b]_{1}-b \otimes[x, a]_{1} \\
& =[x, a]_{1} \otimes b-b \otimes[x, a]_{1}, \quad \text { because } b \in\left(\mathfrak{L}^{+}\right)^{2} \subseteq 3\left(\mathfrak{L}^{-}\right), \\
\Delta_{a}(x) & =\left(x \bullet_{1} a\right) \otimes b-\left(x \bullet \bullet_{1} b\right) \otimes a-a \otimes\left(x \bullet \bullet_{1} b\right)+b \otimes\left(x \bullet_{1} a\right) \\
& =\left(x \bullet_{1} a\right) \otimes b+b \otimes\left(x \bullet_{1} a\right), \quad \text { because } b \in\left(\mathfrak{I}^{+}\right)^{2} \text { and }\left(\mathfrak{Q}^{+}\right)^{3}=\{0\} .
\end{aligned}
$$

Since $a \notin 3\left(\mathfrak{L}^{-}\right)$, then there exists $x_{0} \in \mathfrak{L}$ such that $\left[x_{0}, a\right]_{1} \neq 0$. Consequently $\Delta_{\mathfrak{L}}\left(x_{0}\right) \neq 0$, because $b \notin\left[\mathfrak{L}^{-}, \mathfrak{Q}^{-}\right]_{1}$ implies that $a$ and $b$ are linearly independent. The fact that $a \notin \operatorname{Ann}\left(\mathfrak{L}^{+}\right)$implies that there exists $y_{0} \in \mathfrak{L}$ such that $y_{0} \bullet_{1} a \neq 0$. Thus $\Delta_{a}\left(y_{0}\right) \neq 0$, which proves that $\Delta_{a} \neq 0$. Now, we are going to prove that $r$ satisfies the assertions (1) to (5) of Theorem 4.7. Since $b \in\left(\mathfrak{L}^{+}\right)^{2} \subseteq 3\left(\mathfrak{L}^{-}\right)$, then $\left[r_{12}, r_{13}\right]=\left[r_{12}, r_{23}\right]=\left[r_{13}, r_{23}\right]=0$. Consequently $C(r)=0$, so assertions (1) and (2) of Theorem 4.7 are satisfied. We have

$$
\begin{aligned}
& r_{13} r_{12}=\left(a \bullet \bullet_{1} a\right) \otimes b \otimes b, \\
& r_{12} r_{23}=-b \otimes\left(a \bullet_{1} a\right) \otimes b, \\
& r_{23} r_{13}=b \otimes b \otimes\left(a \bullet_{1} a\right),
\end{aligned}
$$

because $b \in \operatorname{Ann}\left(\mathfrak{L}^{+}\right)$, hence $A(r)=\left(a \bullet \bullet_{1} a\right) \otimes b \otimes b+b \otimes\left(a \bullet_{1} a\right) \otimes b+b \otimes b \otimes\left(a \bullet{ }_{1} a\right)$. It follows that the assertions (3) and (4) of Theorem 4.7 are satisfied because $\left(\mathfrak{L}^{+}\right)^{3}=\{0\}$ and $b \in \operatorname{Ann}\left(\mathfrak{L}^{+}\right) \cap 3\left(\mathfrak{L}^{-}\right)$. Finally, $b \in \operatorname{Ann}\left(\mathfrak{Q}^{+}\right) \cap z\left(\mathfrak{L}^{-}\right)$implies that $\left(\mathbf{M}_{x} \otimes \operatorname{ad}_{y}\right)(r)=0, \forall x, y \in \mathfrak{Q}$, i.e., the assertion (5) of Theorem 4.7 is satisfied. We conclude that $(\mathfrak{L}, ., \Delta)$ is a coboundary Leibniz bialgebra with $\Delta_{\mathfrak{L}} \neq 0$ and $\Delta_{a} \neq 0$.

Second case, we suppose that $\operatorname{Ann}\left(\mathfrak{L}^{+}\right) \cup z\left(\mathfrak{L}^{-}\right)=\mathfrak{L}$, then $\mathfrak{L}=\operatorname{Ann}\left(\mathfrak{L}^{+}\right)+z\left(\mathfrak{L}^{-}\right)$. Since $\left(\mathfrak{L}^{+}\right)^{2} \neq\{0\}$ and $\left[\mathfrak{L}^{-}, \mathfrak{L}^{-}\right]_{1} \neq\{0\}$, it follows that $\operatorname{Ann}\left(\mathfrak{L}^{+}\right) \subsetneq z\left(\mathfrak{L}^{-}\right)$and $z\left(\mathfrak{L}^{-}\right) \subsetneq \operatorname{Ann}\left(\mathfrak{L}^{+}\right)$. Consequently, there exist $v \in z\left(\mathfrak{L}^{-}\right) \backslash \operatorname{Ann}\left(\mathfrak{L}^{+}\right)$and $s \in \operatorname{Ann}\left(\mathfrak{L}^{+}\right) \backslash z\left(\mathfrak{L}^{-}\right)$, so $a=v+s$ is an element of $\mathfrak{L} \backslash\left(\operatorname{Ann}\left(\mathfrak{L}^{+}\right) \cup z\left(\mathfrak{L}^{-}\right)\right)$. If we 
choose $b \in\left(\mathfrak{L}^{+}\right)^{2} \backslash\left[\mathfrak{Q}^{-}, \mathfrak{Q}^{-}\right]_{1}$, then by first case, $r:=a \otimes b-b \otimes a$ defines on $(\mathfrak{L}, ., \Delta)$ a coboundary Leibniz bialgebra structure such that $\Delta_{\mathfrak{Q}} \neq 0$ and $\Delta_{a} \neq 0$.

Remark 5.2. Let $(\mathfrak{L},$.$) be a symmetric Leibniz algebra such that \left(\mathfrak{Q}^{+}\right)^{2} \neq\{0\},\left[\mathfrak{L}^{-}, \mathfrak{L}^{-}\right]_{1} \neq\{0\}$ and $\left(\mathfrak{L}^{+}\right)^{2} \backslash\left[\mathfrak{L}^{-}, \mathfrak{Q}^{-}\right]_{1} \neq \emptyset$. For the Lie algebra $\mathfrak{L}^{-}$, in the proof of the last theorem, we give a new solution of Yang-Baxter equation different from that given in [12].

Proposition 5.3. Let $(\mathfrak{Q},$.$) be a symmetric Leibniz algebra such that \left(\mathfrak{L}^{+}\right)^{2} \subseteq\left[\mathfrak{Q}^{-}, \mathfrak{L}^{-}\right]_{1}$.

(i) If $\left(\mathfrak{Q}^{+}\right)^{2} \neq\left[\mathfrak{Q}^{-}, \mathfrak{Q}^{-}\right]_{1}$, then there exists a coboundary Leibniz bialgebra structure on $(\mathfrak{Q},$.$) such$ that $\Delta_{\mathfrak{Q}} \neq 0$;

(ii) If $\left(\mathfrak{Q}^{+}\right)^{2}=\left[\mathfrak{Q}^{-}, \mathfrak{Q}^{-}\right]_{1}$ and $\operatorname{dim}\left(\mathfrak{Q}^{+}\right)^{2} \geq 2$, then $\mathfrak{Q}^{-}$and $\mathfrak{Q}$ are 2 -nilpotent algebras and there exists a coboundary Leibniz bialgebra structure on $(\mathfrak{Q},$.$) such that \Delta_{\mathfrak{L}} \neq 0$;

(iii) If $\left(\mathfrak{L}^{+}\right)^{2}=\left[\mathfrak{L}^{-}, \mathfrak{L}^{-}\right]_{1}$ and $\operatorname{dim}\left(\mathfrak{L}^{+}\right)^{2}=1$, then $\mathfrak{L}^{-}$and $\mathfrak{L}$ are 2 -nilpotent algebras and there exists a coboundary Leibniz bialgebra structure on $\left(\mathfrak{L}\right.$, .) such that $\Delta_{a} \neq 0$.

Proof. (i) If $\left(\mathfrak{L}^{+}\right)^{2} \neq\left[\mathfrak{L}^{-}, \mathfrak{Q}^{-}\right]_{1}$, then there exist $x_{0}, y_{0} \in \mathfrak{L}$ such that $\left[x_{0}, y_{0}\right]_{1} \notin\left(\mathfrak{L}^{+}\right)^{2}$. Which implies that

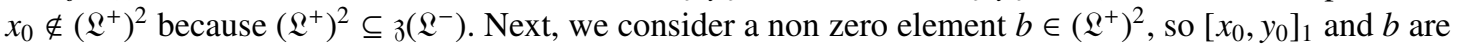
linearly independent. We set $r:=x_{0} \otimes b-b \otimes x_{0}$. If we consider a coproduct $\Delta: \mathfrak{L} \longrightarrow \mathfrak{L} \otimes \mathfrak{L}$ defined by:

$$
\Delta(x)=\left(L_{x} \otimes \mathrm{I}-\mathrm{I} \otimes R_{x}\right)(r), \quad \forall x \in \mathfrak{Q},
$$

then $(\mathfrak{L}, ., \Delta)$ is a coboundary symmetric Leibniz algebra such that $\Delta_{\mathfrak{L}} \neq 0$.

Suppose that $\left(\mathfrak{L}^{+}\right)^{2}=\left[\mathfrak{Q}^{-}, \mathfrak{Q}^{-}\right]_{1}$. Since for all $x, y \in \mathfrak{L}: \quad x . y=[x, y]_{1}+x \bullet 1, y,\left(\mathfrak{L}^{+}\right)^{2} \subseteq z\left(\mathfrak{L}^{-}\right),\left(\mathfrak{L}^{+}\right)^{3}=\{0\}$ and $\left[\mathfrak{Q}^{-}, \mathfrak{Q}^{-}\right] \subseteq \operatorname{Ann}\left(\mathfrak{Q}^{+}\right)$, then $\mathfrak{Q}^{-}$and $\mathfrak{Q}$ are 2 -nilpotent algebras.

(ii) Now, if $\left(\mathfrak{Q}^{+}\right)^{2}=\left[\mathfrak{Q}^{-}, \mathfrak{Q}^{-}\right]_{1}$ and $\operatorname{dim}\left(\mathfrak{Q}^{+}\right)^{2} \geq 2$. Let us consider $x_{0}, y_{0}, b \in \mathfrak{L}$ such that $\left[x_{0}, y_{0}\right]_{1} \neq$ $0, b \in\left(\mathfrak{L}^{+}\right)^{2},\left[x_{0}, y_{0}\right]_{1}$ and $b$ are linearly independent. Consequently, $r:=x_{0} \otimes b-b \otimes x_{0}$ define on $(\mathfrak{L},$. a coboundary symmetric Leibniz algebra such that $\Delta_{\mathfrak{I}} \neq 0$.

(iii) If $\left(\mathfrak{Q}^{+}\right)^{2}=\left[\mathfrak{Q}^{-}, \mathfrak{L}^{-}\right]_{1}$ and $\operatorname{dim}\left(\mathfrak{Q}^{+}\right)^{2}=1$. Then there exist $x_{0}, y_{0}, b \in \mathfrak{L} \operatorname{such}$ that $\left(\mathfrak{Q}^{+}\right)^{2}=\left[\mathfrak{Q}^{-}, \mathfrak{Q}^{-}\right]_{1}=$ $\mathbb{K} b$ and $\left[x_{0}, y_{0}\right]_{1}=b$. Thus, $r:=x_{0} \otimes b-b \otimes x_{0}$ define on $(\mathfrak{L},$.$) a coboundary symmetric Leibniz algebra$ such that $\Delta_{a} \neq 0$.

The following result comes from Theorem 5.1 and Proposition 5.3.

Corollary 5.4. Every symmetric Leibniz algebra $(\mathfrak{L},$.$) , such that \left(\mathfrak{L}^{+}\right)^{2} \neq\{0\}$ and $\left[\mathfrak{L}^{-}, \mathfrak{L}^{-}\right]_{1} \neq\{0\}$, admits a non-trivial coboundary Leibniz bialgebra structure.

\section{Examples of Leibniz bialgebras}

In this section we are going to present some examples of Leibniz algebras which verify either hypothesis of Theorem 5.1 or hypothesis of Proposition 5.3.

Example I. Let $\mathcal{V}$ and $\mathcal{W}$ be two vector spaces. Let us consider $C: \mathcal{V} \times \mathcal{V} \rightarrow \mathcal{W}$ a skew-symmetric bilinear map and $F: \mathcal{V} \times \mathcal{V} \rightarrow \mathcal{W}$ a symmetric bilinear map. On the vector space $\mathfrak{L}:=\mathcal{V} \times \mathcal{W}$ we define the following product:

$$
(v, w) .\left(v^{\prime}, w^{\prime}\right):=C\left(v, v^{\prime}\right)+F\left(v, v^{\prime}\right), \forall v, v^{\prime} \in \mathcal{V}, \forall w, w^{\prime} \in \mathcal{W} .
$$

Simple calculations prove that $(\mathfrak{L},$.$) is a symmetric Leibniz algebra and (\mathfrak{L},$.$) is non-Lie algebra if F \neq 0$. The product $[,]_{1}$ of Lie algebra $\mathfrak{Q}^{-}$(resp. $\bullet_{1}$ of associative commutative algebra $\mathfrak{L}^{+}$) is defined by:

$$
\left[(v, w),\left(v^{\prime}, w^{\prime}\right)\right]_{1}:=C\left(v, v^{\prime}\right) \quad\left(\operatorname{resp} . \quad(v, w) \bullet_{1}\left(v^{\prime}, w^{\prime}\right):=F\left(v, v^{\prime}\right)\right),
$$

$\forall v, v^{\prime} \in \mathcal{V}, \forall w, w^{\prime} \in \mathcal{W}$. It is clear that $\mathfrak{L}, \mathfrak{L}^{-}$and $\mathfrak{L}^{+}$are 2 -nilpotent algebras. We are going to give some examples of Leibniz algebras $\mathfrak{L}:=\mathcal{V} \times \mathcal{W}$ which verify either hypothesis of Theorem 5.1 or hypothesis 
of Proposition 5.3.

1. Consider the case when $\mathcal{V}:=\mathbb{K}^{2}:=\mathbb{K} a+\mathbb{K} X, \mathcal{W}:=\mathbb{K}^{2}:=\mathbb{K} b+\mathbb{K} Y, C_{1}$ and $C_{2}$ be two skewsymmetric bilinear forms on $\mathcal{V}, F_{1}$ and $F_{2}$ be two symmetric bilinear forms on $\mathcal{V}$. Let $C$ (resp. $F$ ) $: \mathcal{V} \times \mathcal{V} \rightarrow \mathcal{W}$ be the skew-symmetric (resp. symmetric) bilinear map defined by:

$$
C(,):=C_{1}(,) b+C_{2}(,) Y\left(\text { resp. } F(,):=F_{1}(,) b+F_{2}(,) Y\right) .
$$

1.1. Suppose that $C_{1}(a, X)=0, C_{2}(a, X)=1, F_{1}(a, a)=1$ and $F_{2}=0$. In this case, $\left[\mathfrak{L}^{-}, \mathfrak{Q}^{-}\right]=\mathbb{K} Y$ and $\left(\mathfrak{L}^{+}\right)^{2}=\mathbb{K} b$. Consequently, $b \in\left(\mathfrak{L}^{+}\right)^{2} \backslash\left[\mathfrak{L}^{-}, \mathfrak{L}^{-}\right]$and $a \in \mathfrak{L} \backslash\left(\operatorname{Ann}\left(\mathfrak{L}^{+}\right) \cup \mathfrak{z}\left(\mathfrak{L}^{-}\right)\right)$. By the proof of Theorem 5.1, $r:=a \otimes b-b \otimes a$ defines a coboundary Leibniz bialgebra $\Delta$ such that $\Delta_{\mathfrak{L}} \neq 0$ and $\Delta_{a} \neq 0$.

1.2. Suppose that $C_{1}(a, X)=1, C_{2}(a, X)=0, F_{1}(a, a)=1$ and $F_{2}=0$. In this case, $\left[\mathfrak{Q}^{-}, \mathfrak{Q}^{-}\right]=\left(\mathfrak{L}^{+}\right)^{2}=$ $\mathbb{K} b$. By the proof of assertion (iii) of Proposition 5.3, $r:=a \otimes b-b \otimes a$ defines a coboundary Leibniz bialgebra $\Delta$ such that $\Delta_{a} \neq 0$. It is easy to see that $\Delta_{\mathfrak{L}}=0$.

2. Now consider the case when $\mathcal{V}:=\mathbb{K}^{3}:=\mathbb{K} a+\mathbb{K} X+\mathbb{K} Z, \mathcal{W}:=\mathbb{K}^{2}=\mathbb{K} b+\mathbb{K} Y, C_{1}$ and $C_{2}$ be two skew-symmetric bilinear forms of $\mathcal{V}, F_{1}$ and $F_{2}$ be two symmetric bilinear forms on $\mathcal{V}$. Let $C$ (resp. $F$ ) $: \mathcal{V} \times \mathcal{V} \rightarrow \mathcal{W}$ be the skew-symmetric (resp. symmetric) bilinear map defined by:

$$
C(,):=C_{1}(,) b+C_{2}(,) Y\left(\text { resp. } F(,):=F_{1}(,) b+F_{2}(,) Y\right) .
$$

Suppose that $C_{1}(a, X)=C_{2}(X, Z)=1, C_{1}(a, Z)=C_{1}(X, Z)=C_{2}(a, X)=C_{2}(a, Z)=0, F_{1}(a, a)=$ $F_{2}(X, X)=1$ and $F_{2}(a, a)=F_{1}(X, X)=0$. In this case, $\left[\mathfrak{L}^{-}, \mathfrak{L}^{-}\right]=\left(\mathfrak{L}^{+}\right)^{2}=\mathcal{W}$. By the proof of assertion (ii) of Proposition 5.3, $r:=X \otimes b-b \otimes X$ defines a coboundary Leibniz bialgebra $\Delta$ such that $\Delta_{\mathfrak{L}} \neq 0$. We have $\Delta_{a}(X)=\left(X \bullet_{1} X\right) \otimes b+b \otimes\left(X \bullet_{1} X\right)=Y \otimes b+b \otimes Y \neq 0$, so $\Delta_{a} \neq 0$.

Example II. Let $\left(\mathfrak{g},[,]_{\mathfrak{g}}\right)$ be a semisimple Lie algebra and $\pi$ its co-adjoint representation. Consider $\mathcal{V}:=\mathbb{K}^{2}:=\mathbb{K} a+\mathbb{K} b$. On the vector space $\mathfrak{L}:=\mathfrak{g} \times \mathfrak{g}^{*} \times \mathcal{V}$ we define the following bilinear product:

$$
(X, f, \alpha a+\beta b) \cdot(Y, h, \lambda a+\gamma b):=\left([X, Y]_{\mathrm{g}}, \pi(X)(h)-\pi(Y)(f)+\alpha h-\lambda f, \alpha \lambda b\right),
$$

$\forall X, Y \in \mathfrak{g}, f, h \in \mathfrak{g}^{*}, \alpha, \beta, \lambda, \gamma \in \mathbb{K}$. Simple calculations prove that $(\mathfrak{L},$.$) is a symmetric Leibniz algebra$ such that $\left(\mathfrak{L}^{+}\right)^{2}=\mathbb{K} b,\left[\mathfrak{L}^{-}, \mathfrak{L}^{-}\right]=\mathfrak{g} \times \mathfrak{g}^{*} \times\{0\}$ which is a perfect Lie algebra and isomorphic to the semidirect product of the abelian Lie algebra $\mathfrak{g}^{*}$ by the Lie algebra $g$ by means of $\pi, 3\left(\mathfrak{L}^{-}\right)=\{0\} \times\{0\} \times \mathbb{K} b$ and $\operatorname{Ann}\left(\mathfrak{L}^{+}\right)=\mathfrak{g} \times \mathfrak{g}^{*} \times \mathbb{K} b$. The proof of Theorem 5.1 shows that $r:=a \otimes b-b \otimes a$ defines a coboundary Leibniz bialgebra $\Delta$ such that $\Delta_{\mathfrak{l}} \neq 0$ and $\Delta_{a} \neq 0$.

Example III. Let us consider the three-dimensional symmetric, solvable and non-nilpotent Leibniz algebra $\mathfrak{L}:=\mathbb{K} X \oplus \mathbb{K} Y \oplus \mathbb{K} Z$ with the product defined by:

$$
Z . Y=Y, \quad Y . Z=-Y, \quad Z . Z=X .
$$

The product $[,]_{1}$ of $\mathfrak{Q}^{-}$is defined by $[Z, Y]_{1}=Y$ and the product $\bullet_{1}$ of $\mathfrak{Q}^{+}$is defined by $Z \bullet{ }_{1} Z=X$. Then $\left[\mathfrak{Q}^{-}, \mathfrak{Q}^{-}\right]=\mathbb{K} Y,\left(\mathfrak{L}^{+}\right)^{2}=\mathbb{K} X, \mathfrak{z}\left(\mathfrak{L}^{-}\right)=\mathbb{K} X$ and $\operatorname{Ann}\left(\mathfrak{L}^{+}\right)=\mathbb{K} X \oplus \mathbb{K} Y$. Consequently $\operatorname{Ann}\left(\mathfrak{L}^{+}\right) \cup \mathfrak{z}\left(\mathfrak{L}^{-}\right)=$ $\mathbb{K} X \oplus \mathbb{K} Y \neq \mathfrak{L}$. Since $Z \in \mathfrak{L} \backslash\left(\operatorname{Ann}\left(\mathfrak{L}^{+}\right) \cup \mathfrak{z}\left(\mathfrak{L}^{-}\right)\right)$and $X \in\left(\mathfrak{L}^{+}\right)^{2} \backslash\left[\mathfrak{L}^{-}, \mathfrak{L}^{-}\right]$. By the proof of Theorem 5.1, $r:=Z \otimes X-X \otimes Z$ defines a coboundary Leibniz bialgebra $\Delta$ such that $\Delta_{\mathfrak{L}} \neq 0$ and $\Delta_{a} \neq 0$. More precisely,

$$
\begin{array}{lll}
\Delta(X)=0, & \Delta(Y)=X \otimes Y-Y \otimes X, & \Delta(Z)=2 X \otimes X ; \\
\Delta_{\mathfrak{L}}(X)=0, & \Delta_{\mathfrak{L}}(Y)=X \otimes Y-Y \otimes X, & \Delta_{\mathfrak{Q}}(Z)=0 ; \\
\Delta_{a}(X)=0, & \Delta_{a}(Y)=0, & \Delta_{a}(Z)=2 X \otimes X .
\end{array}
$$




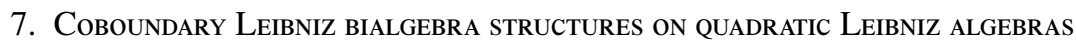

Let $\mathfrak{L}$ be a Leibniz algebra and consider $r=\sum_{i=1}^{n} a_{i} \otimes b_{i}$ with $a_{i}, b_{i} \in \mathfrak{L}$, for all $i \in\{1, \ldots, n\}$. We associate to $r$ a linear map $\mathcal{R}: \mathfrak{R}^{*} \longrightarrow \mathfrak{Q}$ defined by

$$
\mathcal{R}(f):=(f \otimes 1)(r)=\sum_{i=1}^{n} f\left(a_{i}\right) b_{i}, \quad \forall f \in \mathfrak{Q}^{*} .
$$

If $r$ is skew-symmetric, we also have $\mathcal{R}(f)=-\sum_{i=1}^{n} f\left(b_{i}\right) a_{i}$, for all $f \in \mathfrak{Q}^{*}$. In [4] we have studied the class of quadratic Lie (super)algebras with triangular structures. We have proved that for a Lie algebra $\mathfrak{g}$, $r$ is a skew-symmetric solution of the classical Yang-Baxter equation $C(r)=0$ if and only if $\langle f, \mathcal{R}(h)\rangle=$ $-\langle h, \mathcal{R}(f)\rangle$ and the cyclic equation

$$
\sum_{c y c l}\langle f,[\mathcal{R}(h), \mathcal{R}(l)]\rangle=0
$$

hold, for all $f, h, l \in \mathfrak{g}^{*}$ (see [4, Proposition 2.5]). In our present context we may also rewrite conditions of Theorem 4.7 in terms of a map $\mathcal{R}$.

Proposition 7.1. Let $(\mathfrak{L},$.$) be a symmetric Leibniz algebra, r \in \mathfrak{L} \otimes \mathfrak{L}$ a skew-symmetric element and $\mathcal{R}$ the linear map defined by (7.18). We consider the coproduct $\Delta: \mathfrak{L} \longrightarrow \mathfrak{L} \otimes \mathfrak{L}$ defined by:

$$
\Delta(x)=\left(L_{x} \otimes I-I \otimes R_{x}\right)(r), \quad \forall x \in \mathbb{L} .
$$

The triple $(\mathfrak{L}, ., \Delta)$ is a Leibniz bialgebra if and only if these conditions are satisfied:

(1) $\sum_{c y c l}\left(\left\langle a d_{x}^{*}(f),[\mathcal{R}(g), \mathcal{R}(h)]\right\rangle+\left\langle f,\left[\mathcal{R}\left(a d_{x}^{*}(g)\right), \mathcal{R}(h)\right]\right\rangle+\left\langle f,\left[\mathcal{R}(g), \mathcal{R}\left(a d_{x}^{*}(h)\right)\right]\right\rangle\right)=0$,

(2) $\left\langle M_{x}^{*}(h),[\mathcal{R}(f), \mathcal{R}(g)]\right\rangle+\left\langle f,\left[\mathcal{R}(g), \mathcal{R}\left(M_{x}^{*}(h)\right)\right]\right\rangle-\left\langle g,\left[\mathcal{R}(f), \mathcal{R}\left(M_{x}^{*}(h)\right)\right]\right\rangle=0$,

(3) $\left\langle M_{x}^{*}(h), \mathcal{R}(f) \bullet \mathcal{R}(g)\right\rangle+\left\langle f, \mathcal{R}(g) \bullet \mathcal{R}\left(M_{x}^{*}(h)\right)\right\rangle+\left\langle g, \mathcal{R}(f) \bullet \mathcal{R}\left(M_{x}^{*}(h)\right)\right\rangle=0$,

(4) $\left\langle a d_{x}^{*}(h), \mathcal{R}(f) \bullet \mathcal{R}(g)\right\rangle+\left\langle f, \mathcal{R}(g) \bullet \mathcal{R}\left(a d_{x}^{*}(h)\right)\right\rangle+\left\langle g, \mathcal{R}(f) \bullet \mathcal{R}\left(a d_{x}^{*}(h)\right)\right\rangle=0$,

(5) $\left\langle M_{x}^{*}(f), \mathcal{R}\left(a d_{y}^{*}(g)\right)\right\rangle=0$,

for all $x, y \in \mathfrak{L}$ and $f, g, h \in \mathfrak{Q}^{*}$.

Proof. First we rewrite condition (1) of Theorem 4.7 in terms of a map $\mathcal{R}$. We consider always $x, y \in \mathfrak{L}$ and $f, g, h \in \mathfrak{2}^{*}$. Recall that

$$
C(r):=\sum_{i, j=1}^{n}\left(\left[a_{i}, a_{j}\right] \otimes b_{i} \otimes b_{j}+a_{i} \otimes\left[b_{i}, a_{j}\right] \otimes b_{j}+a_{i} \otimes a_{j} \otimes\left[b_{i}, b_{j}\right]\right),
$$

hence

$$
\begin{aligned}
\left(\operatorname{ad}_{x} \otimes \mathrm{I} \otimes\right. & \mathrm{I} \\
+ & \left.\mathrm{I} \otimes \mathrm{ad}_{x} \otimes \mathrm{I}+\mathrm{I} \otimes \mathrm{I} \otimes \mathrm{ad}_{x}\right) C(r)= \\
= & \sum_{i, j=1}^{n}\left(\left[x,\left[a_{i}, a_{j}\right]\right] \otimes b_{i} \otimes b_{j}+\left[x, a_{i}\right] \otimes\left[b_{i}, a_{j}\right] \otimes b_{j}+\left[x, a_{i}\right] \otimes a_{j} \otimes\left[b_{i}, b_{j}\right]\right. \\
& +\left[a_{i}, a_{j}\right] \otimes\left[x, b_{i}\right] \otimes b_{j}+a_{i} \otimes\left[x,\left[b_{i}, a_{j}\right]\right] \otimes b_{j}+a_{i} \otimes\left[x, a_{j}\right] \otimes\left[b_{i}, b_{j}\right] \\
& \left.+\left[a_{i}, a_{j}\right] \otimes b_{i} \otimes\left[x, b_{j}\right]+a_{i} \otimes\left[b_{i}, a_{j}\right] \otimes\left[x, b_{j}\right]+a_{i} \otimes a_{j} \otimes\left[x,\left[b_{i}, b_{j}\right]\right]\right) .
\end{aligned}
$$

We have,

$$
\begin{aligned}
(f \otimes g \otimes h) & \left(\left(\operatorname{ad}_{x} \otimes \mathrm{I} \otimes \mathrm{I}+\mathrm{I} \otimes \operatorname{ad}_{x} \otimes \mathrm{I}+\mathrm{I} \otimes \mathrm{I} \otimes \operatorname{ad}_{x}\right) C(r)\right)= \\
= & -\left\langle\operatorname{ad}_{x}^{*}(f),[\mathcal{R}(g), \mathcal{R}(h)]\right\rangle+\left\langle g,\left[\mathcal{R}\left(\operatorname{ad}_{x}^{*}(f)\right), \mathcal{R}(h)\right]\right\rangle-\left\langle h,\left[\mathcal{R}\left(\operatorname{ad}_{x}^{*}(f)\right), \mathcal{R}(g)\right]\right\rangle \\
& -\left\langle f,\left[\mathcal{R}\left(\operatorname{ad}_{x}^{*}(g)\right), \mathcal{R}(h)\right]\right\rangle+\left\langle\operatorname{ad}_{x}^{*}(g),[\mathcal{R}(f), \mathcal{R}(h)]\right\rangle-\left\langle h,\left[\mathcal{R}(f), \mathcal{R}\left(\operatorname{ad}_{x}^{*}(g)\right)\right]\right\rangle \\
& -\left\langle f,\left[\mathcal{R}(g), \mathcal{R}\left(\operatorname{ad}_{x}^{*}(h)\right)\right]\right\rangle+\left\langle g,\left[\mathcal{R}(f), \mathcal{R}\left(\operatorname{ad}_{x}^{*}(h)\right)\right]\right\rangle-\left\langle\operatorname{ad}_{x}^{*}(h),[\mathcal{R}(f), \mathcal{R}(g)]\right\rangle .
\end{aligned}
$$


Then (1) of Theorem 4.7 is equivalent to the cyclic condition

$$
\sum_{c y c l}\left(\left\langle\operatorname{ad}_{x}^{*}(f),[\mathcal{R}(g), \mathcal{R}(h)]\right\rangle+\left\langle f,\left[\mathcal{R}\left(\operatorname{ad}_{x}^{*}(g)\right), \mathcal{R}(h)\right]\right\rangle+\left\langle f,\left[\mathcal{R}(g), \mathcal{R}\left(\operatorname{ad}_{x}^{*}(h)\right)\right]\right\rangle\right)=0 .
$$

Now we deal we condition (2) of Theorem 4.7. We have

$$
\left(\mathrm{I} \otimes \mathrm{I} \otimes \mathrm{M}_{x}\right) C(r)=\sum_{i, j=1}^{n}\left(\left[a_{i}, a_{j}\right] \otimes b_{i} \otimes\left(x \bullet b_{j}\right)+a_{i} \otimes\left[b_{i}, a_{j}\right] \otimes\left(x \bullet b_{j}\right)+a_{i} \otimes a_{j} \otimes\left(x \bullet\left[b_{i}, b_{j}\right]\right)\right) .
$$

Thus

$$
(f \otimes g \otimes h)\left(\left(\mathrm{I} \otimes \mathrm{I} \otimes \mathrm{M}_{x}\right) C(r)\right)=\left\langle f,\left[\mathcal{R}(g), \mathcal{R}\left(\mathbf{M}_{x}^{*}(h)\right)\right]\right\rangle+\left\langle\mathbf{M}_{x}^{*}(h),[\mathcal{R}(f), \mathcal{R}(g)]\right\rangle-\left\langle g,\left[\mathcal{R}(f), \mathcal{R}\left(\mathrm{M}_{x}^{*}(h)\right)\right]\right\rangle .
$$

Then (2) of Theorem 4.7 is equivalent to the condition

$$
\left\langle\mathrm{M}_{x}^{*}(h),[\mathcal{R}(f), \mathcal{R}(g)]\right\rangle+\left\langle f,\left[\mathcal{R}(g), \mathcal{R}\left(\mathrm{M}_{x}^{*}(h)\right)\right]\right\rangle-\left\langle g,\left[\mathcal{R}(f), \mathcal{R}\left(\mathrm{M}_{x}^{*}(h)\right)\right]\right\rangle=0 .
$$

To deal with condition (3) of Theorem 4.7, we recall that

$$
A(r):=\sum_{i, j=1}^{n}\left(\left(a_{i} \bullet a_{j}\right) \otimes b_{j} \otimes b_{i}-a_{i} \otimes\left(b_{i} \bullet a_{j}\right) \otimes b_{j}+a_{j} \otimes a_{i} \otimes\left(b_{i} \bullet b_{j}\right)\right),
$$

then

$$
\left(\mathrm{I} \otimes \mathrm{I} \otimes \mathrm{M}_{x}\right) A(r)=\sum_{i, j=1}^{n}\left(\left(a_{i} \bullet a_{j}\right) \otimes b_{j} \otimes\left(x \bullet b_{i}\right)-a_{i} \otimes\left(b_{i} \bullet a_{j}\right) \otimes\left(x \bullet b_{j}\right)+a_{j} \otimes a_{i} \otimes\left(x \bullet\left(b_{i} \bullet b_{j}\right)\right)\right) .
$$

Hence

$$
(f \otimes g \otimes h)\left(\left(\mathrm{I} \otimes \mathrm{I} \otimes \mathrm{M}_{x}\right) A(r)\right)=\left\langle f, \mathcal{R}(g) \bullet \mathcal{R}\left(\mathrm{M}_{x}^{*}(h)\right)\right\rangle+\left\langle g, \mathcal{R}(f) \bullet \mathcal{R}\left(\mathrm{M}_{x}^{*}(h)\right)\right\rangle+\left\langle\mathrm{M}_{x}^{*}(h), \mathcal{R}(f) \bullet \mathcal{R}(g)\right\rangle .
$$

Therefore condition (3) of Theorem 4.7 is equivalent to

$$
\left\langle\mathrm{M}_{x}^{*}(h), \mathcal{R}(f) \bullet \mathcal{R}(g)\right\rangle+\left\langle f, \mathcal{R}(g) \bullet \mathcal{R}\left(\mathrm{M}_{x}^{*}(h)\right)\right\rangle+\left\langle g, \mathcal{R}(f) \bullet \mathcal{R}\left(\mathrm{M}_{x}^{*}(h)\right)\right\rangle=0 .
$$

In a similar way we show that (4) of Theorem 4.7 is equivalent to the condition

$$
\left\langle\operatorname{ad}_{x}^{*}(h), \mathcal{R}(f) \bullet \mathcal{R}(g)\right\rangle+\left\langle f, \mathcal{R}(g) \bullet \mathcal{R}\left(\operatorname{ad}_{x}^{*}(h)\right)\right\rangle+\left\langle g, \mathcal{R}(f) \bullet \mathcal{R}\left(\operatorname{ad}_{x}^{*}(h)\right)\right\rangle=0 .
$$

Finally, for condition (5) of Theorem 4.7 we have

$$
\left(\mathrm{M}_{x} \otimes \operatorname{ad}_{y}\right)(r)=\sum_{i=1}^{n}\left(x \bullet a_{i}\right) \otimes\left[y, b_{i}\right]
$$

Hence

$$
(f \otimes g)\left(\left(\mathrm{M}_{x} \otimes \operatorname{ad}_{y}\right)(r)\right)=-\left\langle\operatorname{ad}_{y}^{*}(g), \mathcal{R}\left(\mathrm{M}_{x}^{*}(f)\right)\right\rangle=\left\langle\mathrm{M}_{x}^{*}(f), \mathcal{R}\left(\operatorname{ad}_{y}^{*}(g)\right)\right\rangle .
$$

Then condition (5) of Theorem 4.7 is equivalent to

$$
\left\langle\mathbf{M}_{x}^{*}(f), \mathcal{R}\left(\operatorname{ad}_{y}^{*}(g)\right)\right\rangle=0,
$$

completing the proof.

Now, we assume that $\mathfrak{L}$ is a quadratic Leibniz algebra endowed with an invariant scalar product $B$. Since $B$ is non-degenerate then we may define an isomorphism of vector spaces $\phi: \mathfrak{L} \longrightarrow \mathfrak{L}^{*}$ by

$$
\phi(x):=B(x, .), \quad \forall x \in \mathbb{Q} .
$$

We also define $U:=\mathcal{R} \circ \phi: \mathfrak{L} \longrightarrow \mathfrak{L}$, where $\mathcal{R}$ is the above linear map associated to $r$ expressed by (7.18). 
Proposition 7.2. Let $(\mathfrak{L}$, .) be a quadratic symmetric Leibniz algebra, $r \in \mathfrak{L} \otimes \mathfrak{L}$ a skew-symmetric element and $U$ the map just defined. We consider the coproduct $\Delta: \mathfrak{L} \longrightarrow \mathfrak{L} \otimes \mathfrak{L}$ defined by:

$$
\Delta(x)=\left(L_{x} \otimes I-I \otimes R_{x}\right)(r), \quad \forall x \in \mathfrak{L} .
$$

The triple $(\mathfrak{L}, ., \Delta)$ is a Leibniz bialgebra if and only if these conditions are satisfied: for $a, b, c \in \mathfrak{L}$,

(1) $\sum_{c y c l}([a,[U(b), U(c)]]+[b, U([a, U(c)])]-[c, U([a, U(b)])])=0$,

(2) $[U(a), U(b)]-U([a, U(b)]+[U(a), b]) \in A n n\left(\mathfrak{Q}^{+}\right)$,

(3) $U(a) \bullet U(b)-U(a \bullet U(b)+U(a) \bullet b) \in A n n\left(\mathfrak{Q}^{+}\right)$,

(4) $U(a) \bullet U(b)-U(a \bullet U(b)+U(a) \bullet b) \in z\left(\mathfrak{L}^{-}\right)$,

(5) $U\left(\left[\mathfrak{L}^{-}, \mathfrak{Q}^{-}\right]\right) \subseteq$ Ann $\left(\mathfrak{R}^{+}\right)$or equivalently $U\left(\mathfrak{L}^{+} \bullet \mathfrak{L}^{+}\right) \subseteq z\left(\mathfrak{L}^{-}\right)$.

Proof. First we take care of condition (1) of Proposition 7.1. We consider $a, b, c \in \mathfrak{L}$ and set $f=\phi(a)$, $g=\phi(b), h=\phi(c)$ in $\mathfrak{L}^{*}$. Since $r$ is skew-symmetric, $\langle f, \mathcal{R}(g)\rangle=-\langle g, \mathcal{R}(f)\rangle$ and so $B(a, U(b))=$ $-B(b, U(a))=-B(U(a), b)$. We have for $x, y \in \mathfrak{L}$

$$
\operatorname{ad}_{x}^{*}(\phi(a))(y)=-\phi(a)([x, y])=-B(a,[x, y])=B([x, a], y)=\phi([x, a])(y) .
$$

So

$$
\begin{aligned}
\sum_{c y c l}( & \left.\left\langle\operatorname{ad}_{x}^{*}(f),[\mathcal{R}(g), \mathcal{R}(h)]\right\rangle+\left\langle f,\left[\mathcal{R}\left(\operatorname{ad}_{x}^{*}(g)\right), \mathcal{R}(h)\right]\right\rangle+\left\langle f,\left[\mathcal{R}(g), \mathcal{R}\left(\operatorname{ad}_{x}^{*}(h)\right)\right]\right\rangle\right)= \\
& =\sum_{c y c l}\left(\left\langle\operatorname{ad}_{x}^{*}(\phi(a)),[U(b), U(c)]\right\rangle+\left\langle\phi(a),\left[R\left(\operatorname{ad}_{x}^{*}(\phi(b))\right), U(c)\right]\right\rangle+\left\langle\phi(a),\left[U(b), R\left(\operatorname{ad}_{x}^{*}(\phi(c))\right)\right]\right\rangle\right) \\
& =\sum_{c y c l}(-B(a,[x,[U(b), U(c)]])+B(a,[U([x, b]), U(c)])+B(a,[U(b), U([x, c])])) \\
& =\sum_{c y c l}(B([a,[U(b), U(c)]], x)+B(U([a, U(c)]),[x, b])-B(U([a, U(b)]),[x, c])) \\
& =\sum_{c y c l}(B([a,[U(b), U(c)]]+[b, U([a, U(c)])]-[c, U([a, U(b)])], x)) .
\end{aligned}
$$

Since $B$ is non-degenerate, then (1) of Proposition 7.1 is equivalent to the cyclic condition

$$
\sum_{c y c l}([a,[U(b), U(c)]]+[b, U([a, U(c)])]-[c, U([a, U(b)])])=0 .
$$

Now we deal with condition (2) of Proposition 7.1. We have for $x, y \in \mathfrak{L}$

$$
\mathbf{M}_{x}^{*}(\phi(a))(y)=\phi(a)(x \bullet y)=B(a, x \bullet y)=B(x \bullet a, y)=\phi(x \bullet a)(y) .
$$

Hence

$$
\begin{aligned}
\left\langle\mathrm{M}_{x}^{*}(h),[\mathcal{R}(f), \mathcal{R}(g)]\right\rangle+\left\langle f,\left[\mathcal{R}(g), \mathcal{R}\left(\mathrm{M}_{x}^{*}(h)\right)\right]\right\rangle-\left\langle g,\left[\mathcal{R}(f), \mathcal{R}\left(\mathrm{M}_{x}^{*}(h)\right)\right]\right\rangle= \\
=\left\langle\mathrm{M}_{x}^{*}(\phi(c)),[U(a), U(b)]\right\rangle+\left\langle\phi(a),\left[U(b), R\left(\mathrm{M}_{x}^{*}(\phi(c))\right)\right]\right\rangle-\left\langle\phi(b),\left[U(a), R\left(\mathrm{M}_{x}^{*}(\phi(c))\right)\right]\right\rangle \\
=B(x \bullet c,[U(a), U(b)])+B(a,[U(b), U(x \bullet c)])-B(b,[U(a), U(x \bullet c)]) \\
=B(x, c \bullet[U(a), U(b)])-B(U([a, U(b)]) \bullet c, x)-B(U([U(a), b]) \bullet c, x) \\
=B(([U(a), U(b)]-U([a, U(b)]+[U(a), b])) \bullet c, x) .
\end{aligned}
$$

Since $B$ is non-degenerate, condition (2) of Proposition 7.1 is

$$
([U(a), U(b)]-U([a, U(b)]+[U(a), b])) \bullet c=0, \quad \forall c \in \mathbb{R},
$$

means,

$$
[U(a), U(b)]-U([a, U(b)]+[U(a), b]) \in \operatorname{Ann}\left(\mathfrak{L}^{+}\right) .
$$


In relation to condition (3) of Proposition 7.1 we have

$$
\begin{aligned}
\left\langle\mathrm{M}_{x}^{*}(h),\right. & \mathcal{R}(f) \bullet \mathcal{R}(g)\rangle+\left\langle f, \mathcal{R}(g) \bullet \mathcal{R}\left(\mathrm{M}_{x}^{*}(h)\right)\right\rangle+\left\langle g, \mathcal{R}(f) \bullet \mathcal{R}\left(\mathrm{M}_{x}^{*}(h)\right)\right\rangle= \\
& =\left\langle\mathrm{M}_{x}^{*}(\phi(c)), U(a) \bullet U(b)\right\rangle+\left\langle\phi(a), U(b) \bullet \mathcal{R}\left(\mathrm{M}_{x}^{*}(\phi(c))\right)\right\rangle+\left\langle\phi(b), U(a) \bullet \mathcal{R}\left(\mathrm{M}_{x}^{*}(\phi(c))\right)\right\rangle \\
& =B(x \bullet c, U(a) \bullet U(b))+B(a, U(b) \bullet U(x \bullet c))+B(b, U(a) \bullet U(x \bullet c)) \\
& =B(x, c \bullet(U(a) \bullet U(b)))-B(U(a \bullet U(b)) \bullet c, x)-B(U(U(a) \bullet b) \bullet c, x) \\
& =B(U(a) \bullet U(b)-U(a \bullet U(b)+U(a) \bullet b) \bullet c, x) .
\end{aligned}
$$

Since $B$ is non-degenerate, condition (3) of Proposition 7.1 is

$$
(U(a) \bullet U(b)-U(a \bullet U(b)+U(a) \bullet b)) \bullet c=0, \quad \forall c \in \mathbb{L},
$$

meaning that,

$$
U(a) \bullet U(b)-U(a \bullet U(b)+U(a) \bullet b) \in \operatorname{Ann}\left(\mathfrak{Q}^{+}\right) .
$$

In relation to condition (4) of Proposition 7.1 we have

$$
\begin{aligned}
\left\langle\operatorname{ad}_{x}^{*}(h), \mathcal{R}(f) \bullet \mathcal{R}(g)\right\rangle+\left\langle f, \mathcal{R}(g) \bullet \mathcal{R}\left(\operatorname{ad}_{x}^{*}(h)\right)\right\rangle+\left\langle g, \mathcal{R}(f) \bullet \mathcal{R}\left(\operatorname{ad}_{x}^{*}(h)\right)\right\rangle= \\
\quad=\left\langle\operatorname{ad}_{x}^{*}(\phi(c)), U(a) \bullet U(b)\right\rangle+\left\langle\phi(a), U(b) \bullet \mathcal{R}\left(\operatorname{ad}_{x}^{*}(\phi(c))\right)\right\rangle+\left\langle\phi(b), U(a) \bullet \mathcal{R}\left(\operatorname{ad}_{x}^{*}(\phi(c))\right)\right\rangle \\
\quad=B([x, c], U(a) \bullet U(b))+B(a, U(b) \bullet U([x, c]))+B(b, U(a) \bullet U([x, c])) \\
\quad=B(x,[c, U(a) \bullet U(b)])-B([c, U(a \bullet U(b))], x)-B([c, U(U(a) \bullet b)], x) \\
\quad=B([c, U(a) \bullet U(b)-U(a \bullet U(b)+U(a) \bullet b)], x) .
\end{aligned}
$$

Since $B$ is non-degenerate, condition (4) of Proposition 7.1 is

$$
[c, U(a) \bullet U(b)-U(a \bullet U(b)+U(a) \bullet b)]=0, \quad \forall c \in \mathfrak{L},
$$

which is,

$$
U(a) \bullet U(b)-U(a \bullet U(b)+U(a) \bullet b) \in z\left(\mathfrak{Q}^{-}\right) .
$$

We observe that the pair of conditions (7.20) and (7.21) together, is equivalent to have

$$
U(a) \bullet U(b)-U(a \bullet U(b)+U(a) \bullet b) \in \operatorname{Ann}(\mathfrak{L}) .
$$

Finally, for condition (5) of Proposition 7.1 we have

$$
\left\langle\mathrm{M}_{x}^{*}(f), \mathcal{R}\left(\operatorname{ad}_{y}^{*}(g)\right)\right\rangle=\left\langle\mathrm{M}_{x}^{*}(\phi(a)), \mathcal{R}\left(\operatorname{ad}_{y}^{*}(\phi(b))\right)\right\rangle=B(x \bullet a, U([y, b]))=B(x, a \bullet U([y, b])),
$$

Since $B$ is non-degenerate, condition (5) of Proposition 7.1 is equivalent to

$$
a \bullet U([y, b])=0, \quad \forall a \in \mathfrak{L},
$$

that is, $U\left(\left[\mathfrak{Q}^{-}, \mathfrak{Q}^{-}\right]\right) \subseteq \operatorname{Ann}\left(\mathfrak{Q}^{+}\right)$. Similar, we can show that condition (5) of Proposition 7.1 is equivalent to $U\left(\mathfrak{Q}^{+} \bullet \mathfrak{Q}^{+}\right) \subseteq z\left(\mathfrak{Q}^{-}\right)$, so the proof is complete.

Proposition 7.3. Assume that $\mathfrak{I}$ is a coboundary Leibniz bialgebra such that the co-multiplication on $\mathfrak{L}$ is defined by a skew-symmetric element $r \in \mathfrak{L} \otimes \mathfrak{L}$. Then the multiplication on $\mathfrak{L}^{*}$ defined by (3.4) is determined explicitly by

$$
f \star g=-f \circ R_{\mathcal{R}(g)}-g \circ L_{\mathcal{R}(f)}, \quad \forall f, g \in \mathfrak{Q}^{*},
$$

where $\mathcal{R}$ is the linear map associated to $r$ defined by (7.18). 
Proof. Set $f, g \in \mathfrak{L}^{*}$. For $x \in \mathfrak{L}$ we have

$$
\begin{aligned}
\langle f \star g, x\rangle=\langle f \otimes g, \Delta(x)\rangle & =\left\langle f \otimes g, \sum_{i=1}^{n}\left(\left(x . a_{i}\right) \otimes b_{i}-a_{i} \otimes\left(b_{i} \cdot x\right)\right)\right\rangle \\
& =\sum_{i=1}^{n}\left(f\left(x \cdot a_{i}\right) g\left(b_{i}\right)-f\left(a_{i}\right) g\left(b_{i} \cdot x\right)\right) \\
& =f\left(x . \sum_{i=1}^{n} g\left(b_{i}\right) a_{i}\right)-g\left(\sum_{i=1}^{n} f\left(a_{i}\right) b_{i} . x\right) \\
& =-f(x \cdot \mathcal{R}(g))-g(\mathcal{R}(f) \cdot x) \\
& =-f \circ R_{\mathcal{R}(g)}(x)-g \circ L_{\mathcal{R}(f)}(x),
\end{aligned}
$$

as required.

Proposition 7.4. Assume that $\mathfrak{Q}$ is a quadratic coboundary Leibniz bialgebra such that the co-multiplication on $\mathfrak{L}$ is defined by a skew-symmetric element $r \in \mathfrak{L} \otimes \mathfrak{L}$. The bilinear map $(.)_{*}: \mathfrak{Q} \times \mathfrak{Q} \longrightarrow \mathfrak{L}$ defined by

$$
(a . b)_{*}=\phi^{-1}(\phi(a) \star \phi(b)), \quad \forall a, b \in \mathbb{L},
$$

where $\phi$ is determined by (7.19), can be rewritten as

$$
(a \cdot b)_{*}=-U(b) \cdot a-b \cdot U(a), \quad \forall a, b \in \mathfrak{L},
$$

where $U=\mathcal{R} \circ \phi$, and $\mathcal{R}$ is the linear map associated to $r$ defined by (7.18). Furthermore, $\mathfrak{Q}$ equipped with the multiplication defined by (7.23) is a Leibniz algebra.

Proof. Set $a, b \in \mathfrak{L}$. There exist $f, g \in \mathfrak{L}^{*}$ such that $f=\phi(a)$ and $g=\phi(b)$, respectively. From (7.22), since $B$ is invariant and symmetric, we get for any $x \in \mathfrak{L}$

$$
\begin{aligned}
\langle\phi(a) \star \phi(b), x\rangle & =-\phi(a) \circ R_{\mathcal{R}(\phi(b))}(x)-\phi(b) \circ L_{\mathcal{R}(\phi(a))}(x) \\
& =-\phi(a) \circ R_{U(b)}(x)-\phi(b) \circ L_{U(a)}(x) \\
& =-B(a, x \cdot U(b))-B(b, U(a) \cdot x) \\
& =B(-U(b) \cdot a-b \cdot U(a), x),
\end{aligned}
$$

so $\phi^{-1}(\phi(a) \star \phi(b))=-U(b) . a-b . U(a)$, because $\phi$ is an isomorphism of vector spaces. From (7.23) we get for $a, b, c \in \mathfrak{Q}$,

$$
\left(a .(b . c)_{*}\right)_{*}=\phi^{-1}(\phi(a) \star(\phi(b) \star \phi(c))) .
$$

Then the Leibniz identity of $\mathfrak{L}$ provided with the multiplication given by (7.23) comes easily from the correspondent identity on Leibniz algebra $\left(\mathfrak{Q}^{*}, \star\right)$, completing the proof.

Let $(\mathfrak{L}, ., B)$ be a quadratic symmetric Leibniz algebra with a derivation $D: \mathfrak{L} \longrightarrow \mathfrak{Q}$ which is invertible and $B$-skew-symmetric (i.e., $B(D(x), y)=-B(x, D(y))$, for $x, y \in \mathfrak{Q})$. Then $U:=D^{-1}$ satisfies conditions (1) to (5) of Proposition 7.2. Consequently, $\mathcal{R}=U \circ \phi^{-1}=D^{-1} \circ \phi^{-1}=(\phi \circ D)^{-1}$ define a Leibniz bialgebra $(\mathfrak{L}, ., \Delta)$ with the coproduct defined by $\Delta(x)=\left(L_{x} \otimes \mathrm{I}-\mathrm{I} \otimes R_{x}\right)(r), \forall x \in \mathfrak{L}$, where $r$ is associated to $\mathcal{R}$. We can construct a quadratic symmetric Leibniz algebra with an invertible skew-symmetric derivation (classical way).

Lemma 7.5. Let $(A,$.$) be a symmetric Leibniz algebra. The vector space T_{0}^{*}:=A \oplus A^{*}$ provided with the product given by

$$
(x+f) \odot(y+g)=x \cdot y+\left(R_{A}\right)^{*}(y)(f)+\left(L_{A}\right)^{*}(x)(g),
$$

and the bilinear form $B$ defined by

$$
B(x+f, y+g)=g(x)+f(y),
$$


whenever $x, y \in A, f, g \in A^{*}$, is a quadratic symmetric Leibniz algebra. Let $D$ be an invertible derivation of $A$. Denote by $D^{*}: A^{*} \longrightarrow A^{*}$ the linear map defined by $D^{*}(f)(x)=-f(D(x)), \forall x \in A, f \in A^{*}$ (that is, $D^{*}=-{ }^{t} D$, where ${ }^{t} D$ is the transpose of $\left.D\right)$. Therefore the linear map $\delta: T_{0}^{*} \longrightarrow T_{0}^{*}$ defined by

$$
\delta(x+f):=D(x)+D^{*}(f)=D(x)-{ }^{t} D(f), \quad \forall x \in A, f \in A^{*},
$$

is an invertible skew-symmetric derivation of $T_{0}^{*}$.

Proof. Doing easy calculations we show that $\delta$ is a derivation of $T_{0}^{*}$. Let $x+f, y+g \in T_{0}^{*}$, then

$$
\begin{aligned}
B(\delta(x+f), y+g) & =B\left(D(x)+D^{*}(f), y+g\right) \\
& =D^{*}(f)(y)+g(D(x)) \\
& =-f(D(y))-D^{*}(g)(x) \\
& =-B\left(x+f, D(y)+D^{*}(g)\right) \\
& =-B(x+f, \delta(y+g)),
\end{aligned}
$$

meaning that the derivation $\delta$ is $B$-skew-symmetric.

Example 7.6. Let $(\mathfrak{Q},$.$) be a symmetric Leibniz algebra, consider the associative algebra A_{n}=X \mathbb{K}[X] /\left(X^{(n+1)}\right)$, with $n \in \mathbb{N}$. The vector space $A=\mathfrak{L} \otimes_{\mathbb{K}} A_{n}$ with the multiplication defined by: $\forall x, y \in \mathfrak{L}, \forall p, q \in \mathbb{N}$, $\left(x \otimes \overline{t^{p}}\right) \cdot\left(y \otimes \overline{t^{q}}\right)=x . y \otimes \overline{t^{p+q}}$, is a symmetric Leibniz algebra. The linear map $D: A \longrightarrow A$ defined by $D\left(x \otimes \overline{t^{p}}\right)=p\left(x \otimes \overline{t^{p}}\right), \forall x \in \mathfrak{L}, \forall p \in\{1, \ldots, n\}$, is an invertible derivation of $A$. The linear map $\delta: T_{0}^{*} \longrightarrow T_{0}^{*}$ given by $\delta(x+f)=D(x)-f \circ D, \forall x \in A, f \in A^{*}$, is an invertible skew-symmetric derivation of $T_{0}^{*}$.

\section{ACKNOWLEDGEMENT}

The authors are grateful to the referee for its helpful suggestions to the original manuscript.

\section{REFERENCES}

[1] M. Aguiar, Infinitesimal Hopf algebras. New trends in Hopf algebra theory (La Falda, 1999), 1 - 29, Contemp. Math., 267, Amer. Math. Soc., Providence, RI, 2000.

[2] M. Aguiar, On the associative analog of Lie bialgebras. J. Algebra 244 (2001), no. 2, 492 - 532.

[3] D.W. Barnes, On Levi's theorem for Leibniz algebras, Bull. Aust. Math. Soc. 86 (2012), no. 2, $184-185$.

[4] E. Barreiro and S. Benayadi, Quadratic symplectic Lie superalgebras and Lie bi-superalgebras, J. Algebra 321 (2009), 582 608.

[5] S. Benayadi and M. Boucetta, Special bi-invariant linear connections on Lie groups and finite dimensional Poisson structures, Differential Geom. Appl. 36 (2014), 66 - 89.

[6] S. Benayadi and S. Hidri, Quadratic Leibniz algebras, J. Lie Theory, 24 (2014), no. 3, 737 - 759.

[7] J.M. Casas, M. Ladra, B.A. Omirove and I.A. Karimjanov, Classification of solvable Leibniz algebras with naturally gradedfiliform nilradical, Linear Algebra and Appl. 438 (2013), no. 7, 2973 - 3000.

[8] V. Chari and A. Pressley, A guide to quantum groups, Cambridge University Press, 1994.

[9] S. Covez, The local integration of Leibniz algebras, Ann. Inst. Fourier (Grenoble) 63 (2013), no. 1, $1-35$.

[10] C. Cuvier, Algèbres de Leibnitz: Définition, propriétées, Ann. Sci. École Norm. Sup. (4) 27 (1994), 1 - 45.

[11] V.G. Drinfeld, Quantum groups, Proc. ICM 1986, AMS 1 (1987), 798 - 820, Hamilton-Lie groups, Lie bialgebras and the geometric meaning of the Yang-Baxter equations, Dokl. Akad. Nauk SSSR (1982).

[12] J. Feldvoss, Existence of triangular Lie bialgebra structures, J. Pure Appl. Algebra, 134 (1999), 1 - 14.

[13] A. Fialowski, L. Magnin and A. Mandan, About Leibniz cohomology and deformations of Lie algebras, J. Algebra 383 (2013), $63-77$.

[14] M. Geoffrey and Y. Gaywalee, Leibniz algebras and Lie algebras, SIGMA Symmetry Integrability Geom. Methods Appl. 9 (2013), Paper 063, 10 pp.

[15] S. Gòmez-Vidal, A. Kh. Khudoyberdiyev and B.A. Omirov, Some remarks on semisimple Leibniz algebras, J. Algebra 410 (2014), $526-540$.

[16] A. Hedges and E. Stitzinger, More on the Frattini subalgebra of a Leibniz algebra, arXiv:1206.5707v2 [math.RA] (2013).

[17] S. A. Joni and G. C. Rota, Coalgebras and bialgebras in combinatorics, Stud. Appl. Math. 61 (1979), no. 2, 93 - 139. Reprinted in Gian-Carlo Rota on Combinatorics: Introductory papers and commentaries (Joseph P.S. Kung, Ed.), Birkhäuser, Boston (1995). 
[18] J-L Loday, Une version non commutative des algèbres de Lie: les algèbres de Leibniz. (French) [A noncommutative version of Lie algebras: the Leibniz algebras], Enseign. Math. (2) 39 (1993), no. 3-4, 269 - 293.

[19] W. Michaelis, Lie coalgebras, Adv. in Math. 38 (1980), no. 1, 1 - 54.

[20] A. Rezaei-Aghdam, GH. Haghighatdoost and L. Sedghi-Ghadim, Leibniz bialgebras, arXiv:1401.6845v1 [math-ph] (2014).

Elisabete Barreiro, CMUC, Department of Mathematics, University of Coimbra, Apartado 3008, 3001-454 Coimbra, PortuGAL E-mail address: MEFB@MAT.UC.PT

Saïd Benayadi, Université de Lorraine, Laboratoire IECL, CNRS UMR 7502, Ile du Saulcy, 57045 Metz cedex 01, France E-mail address: SAID.BENAYADI@UNIV-LORRAINE.FR 